\title{
Opinion Behavior Analysis in Social Networks under the Influence of Coopetitive Media
}

\author{
Dong Xue, Member, IEEE, Sandra Hirche, Senior Member, IEEE, and Ming Cao, Senior Member, IEEE
}

\begin{abstract}
Both interpersonal communication and media contact are important information sources and play a significant role in shaping public opinions of large populations. In this article, we investigate how the opinion-forming process evolves over social networks under the media influence. In addition to being affected by the opinions of their connected peers, the media cooperate and/or compete mutually with each other. Networks with mixed cooperative and competitive interactions are said to be coopetitive. In this endeavor, a novel mathematical model of opinion dynamics is introduced, which captures the information diffusion process under consideration, makes use of the community-based network structure, and takes into account personalized biases among individuals in social networks. By employing port-Hamiltonian system theory to analyze the modeled opinion dynamics, we predict how public opinions evolve in the long run through social entities and find applications in political strategy science. A key technical observation is that as a result of the port-Hamiltonian formulation, the mathematical passivity property of individuals' self-dynamics facilitates the convergence analysis of opinion evolution. We explain how to steer public opinions towards consensus, polarity, or neutrality, and investigate how an autocratic media coalition might emerge regardless of public views. We also assess the role of interpersonal communication and media exposure, which in itself is an essential topic in mathematical sociology.
\end{abstract}



Social networks, media influence, opinion-forming processes, social corruption, port-Hamiltonian representation.

\section{INTRODUCTION}

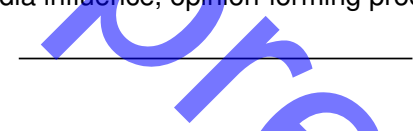

OCIAL entities share and aggregate thoughts, ideas, feelings, experience, and observations over social networks, and generate new thoughts, ideas, feelings, experiences, and observations at the same time. In social science, numerous efforts have been directed towards understanding the relationship between individual behaviors and social interaction among actors [1] $-[3]$.

The conventional modeling methods of social networks often postulate that a social actor communicates directly with other connected peers. In practice, however, interaction in the form of communication or observation between social actors occurs not directly but rather through some intermediates or a shared environment [4]. An analogy can be found in biological systems [5], where bacteria produce, release, and measure signaling molecules (known as autoinducers) which disseminate in the environment, influencing population coordination and bacterial infection processes. This mechanism, termed quorum-sensing transitions [6] (see Fig. 1(a)), appears to be "déjà vu" in social science. In reality, aside from actorto-actor communication, the messages delivered either through the traditional media, e.g., TV, radio, and newspaper, or trendy socio-technical platforms, e.g., blogs, Facebook, and Twitter are also important sources of formulating and changing people's attitudes towards relevant topics. For instance, according to a study of the impact of media bias on US voting [7], Fox News, a cable and satellite TV news channel, helps Republicans gain an estimated 3 to 8 percent of additional votes between 1996 and 2000. Meanwhile, media are affected to different extents by their audiences and other presses. Nowadays, viewers no longer passively receive messages but may behave proactively. Another significant class of social networks where quorum sensing can take place is the system of governance in which a small deliberative group assembles in a large organization. Those elected or appointed members coming from different interest communities are authorized to deal with issues in particular domains. Notable examples include committees in universities or enterprises, School Boards in public school districts, Boards of Directors in the organizations, and elected officials and standing policy bodies in the Congress [8]. The quorum-sensing communication architecture (see Fig. 1(b)], despite arising from different social contexts, motivates the in-depth study of opinion dynamics. Along with this line of research, most literature focuses on the understanding of the opinion-forming processes under the exogenous influence which is often modeled by a constant [9], time-varying [10], or noisy signal [11]. Other works [10], [12] take into account the importance of media on audiences and suggest using a bounded confidence model (BCM) to formulate the communication links. The truncation effect of $\mathrm{BCM}$, however, prevents the use of commonly available tools for analyzing dynamical systems.

The recent developments in network science uncover that many network-embedded dynamical systems might appear to persistent disagreement or even cleavage [1], not just unanimous behavior [13]. Among others, one response to

- D. Xue and M. Cao are with the Faculty of Science and Engineering, University of Groningen, Groningen 9747 AG, The Netherlands. E-mail: dongtonyxue@gmail.com

- D. Xue and S. Hirche are with the Chair of Information-Oriented Control, Technische Universität München, D-80209 München, Germany. 


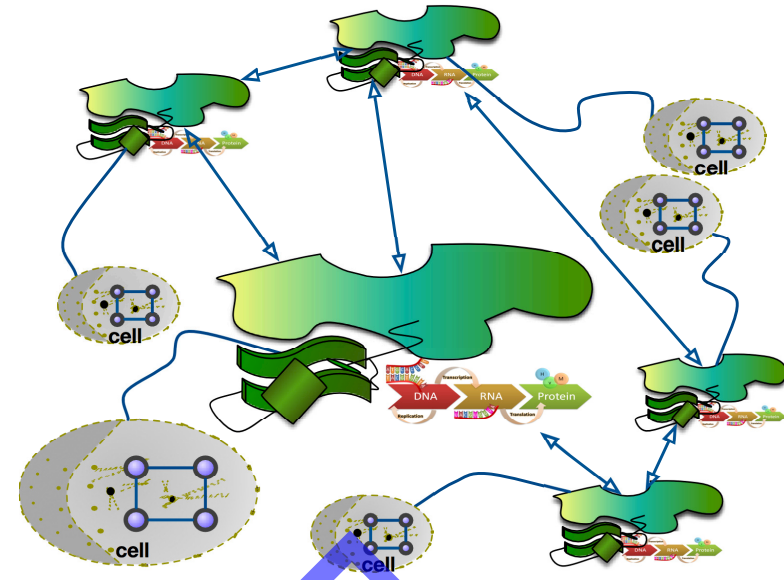

(a)

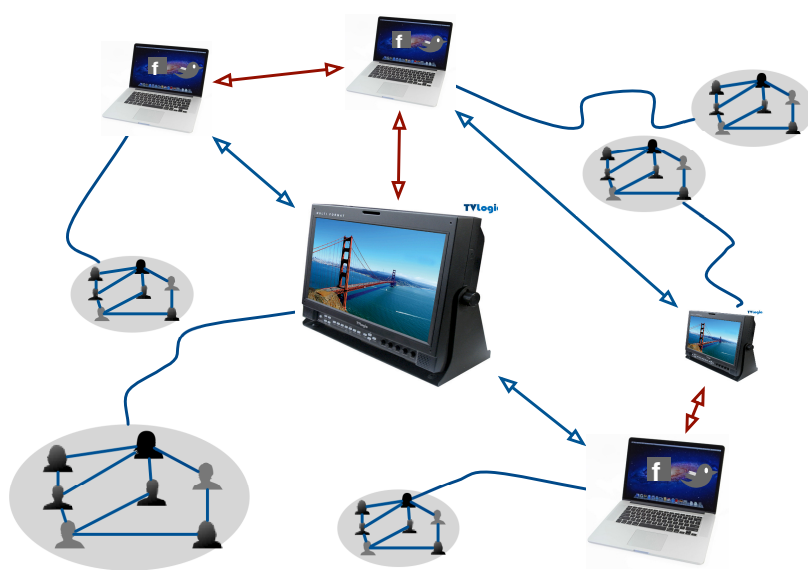

(b)

Fig. 1: A schematic representation of the networks. (a). A bacterial quorum-sensing network. (b). A social network with cooperative (in red) and competitive (in blue) interactions.

opinion separation is the emergence of antagonistic relationships between social entities including competition, distrust, rebellion, and betrayal [14]. In comparison to manufacturing consent in cooperative networks, numerous efforts have been directed toward investigating mechanisms for polarization on social networks with adversarial behaviors [3]. Meanwhile, competition happens in media industries for political or commercial purposes, e.g., to attract the audience [12], motivating to re-examine the understanding of opinion dynamics in cooperative scenarios. The aforecited literature, however, primarily focuses on understanding the connection between the opinion evolution and the interactions via communication networks. In this article, we emphasize the fundamental importance of individuals' dynamical properties on opinion formation. This is rooted in the psychological fact that social entities, primarily humans, are not utterly rational and even ordinarily intentional [15]. Moreover, individual diversity has a significant impact on the opinion formulation of social entities. Individuals, who live in the same community, may still have different educational experience, dissenting political views, and contrasting aesthetic standards, substantially affecting their decision making. The existing methods including the gauge transformation [16] and lifting approach |17| base upon the discrete-time DeGroot-type model or the continuoustime Abelson-type model, and thus are only valid in the absence of self-dynamics. Therefore, there is a great need for constructing an appropriate methodology to study the opinion formation problem of heterogeneous agents with selfcognition.

Contributions In this work, we elaborate on the study of opinion formation problems in social networks under the influence of media. A new mathematical model involving local dynamics and interaction structures is introduced to describe the evolving properties of opinion dynamics. On the one hand, the modeling framework shows social entities tend to timely update their beliefs through the received messages from neighboring peers and social media. In particular, the media competition is embodied explicitly by assigning negative signs to the edges corresponding to antagonistic interactions on graphs. On the other hand, the developed mathematical formulation emphasizes the existence and importance of the individual dynamics in the opinion evolution process. Motivated by the market segmentation and business concentration in media industries, we analyze the dynamical properties of the developed model of opinion dynamics at the level of community architectures. By employing port-Hamiltonian (PH) formulation to represent the opinion dynamics, we can gain insight into how agents behave confronting a massive body of external influences. In connection with the concept of internalization in psychological and sociological studies [18], the PH formulation explains the external information or message flow through social entities without relying on detailed social psychological descriptions. Additionally, the convergence analysis follows naturally from the PH system theory, underlining the collective effect of local dynamic natures and topological constraints on shaping public opinion. Furthermore, the sociological postulate "iron law of oligarchy" |19| motivates our further investigation on how media dominate the opinion-pooling process in autocratic societies. From a control-theoretic perspective, we explore the intrinsic mechanisms and ruling strategies of dominant groups controlling outcomes of social systems, which may lead to new ideas for policy intervention or prevention.

Organization The remainder of the article is organized as follows. Section 2 develops the mathematical model of opinion dynamics through interpersonal communication and media influence and introduces some preliminary properties of this system. Using networks based on communities, we study in Section 3 the fundamental properties of the developed framework and provide sufficient conditions for diverse opinion forming patterns. Section 4 presents some necessary modeling interpretations and steady-state analysis. Some numerical examples are given in Section 5 to illustrate the results. Finally, we conclude the article in Section VI together with some discussions on future works.

Notations Let $\left(\mathbb{R}_{\geq 0}\right) \mathbb{R}$ and $\left(\mathbb{C}_{\geq 0}\right) \mathbb{C}$ be the set of (non-negative) real and (non-negative) complex numbers, respectively. Vector $\mathbf{1}_{n}\left(\mathbf{0}_{n}\right)$ and matrix $\boldsymbol{I}_{n}$ represent respectively the $n$-dimensional column vector of all ones (zeros) and the $n \times n$ 
identity matrix with appropriate dimensions. The notation $|a|$ and sgn $a$ denote the absolute value and sign pattern of a scalar $a$, receptively. Moreover, $|\boldsymbol{x}|$ and $\|\boldsymbol{x}\|$ are the entry-wise absolute value and the 2-norm of a vector $\boldsymbol{x}=\left(x_{1}, \ldots, x_{n}\right)$, respectively. The notation $\boldsymbol{M} \succ 0(\boldsymbol{M} \succeq 0)$ represents that matrix $\boldsymbol{M}$ is positive (non-negative) definite. The spectrum of a square matrix $M$ is written by $\operatorname{sp}(\boldsymbol{M})$. Let $\iota$ be the imaginary unite, i.e., $\iota^{2}=-1$ and $\iota \mathbb{R}$ represent the imaginary axis containing the number zero. An eigenvalue of a matrix is semi-simple if it possesses equal algebraic and geometric multiplicities.

Throughout the article, we shall use the terminology "agents" for all social entities living on a network and model them by nodes in a graph. Among others, we shall refer to "media" or "leaders" as the social entities who have predominance in social activities, which correspond to the dominant nodes in the graph. We shall adopt "actors" to describe the entities that amount to the other ordinary nodes in the graph.

\section{Problem formulation}

\subsection{Basic Notions From Graph Theory}

A signed graph is denoted by $\mathcal{G}=(\mathbb{V}, \mathbb{E})$ where $\mathbb{V}=\{1, \ldots, n\}$ stands for the set of nodes and $\mathbb{E} \subseteq \mathbb{V} \times \mathbb{V}$ is a set of edges. Define the adjacency matrix by $\boldsymbol{A}=\left[a_{i j}\right] \in \mathbb{R}^{n \times n}$ such that an element $(j, i)$ of $\mathbb{E}$ indicates that there exists a directed edge from node $j$ to $i$ with the coupling weight $a_{i j} \neq 0$. Throughout the article, we confine ourselves to graphs that have no self-loops (i.e., $a_{i i}=0, \forall i \in \mathbb{V}$ ) and are digon sign-symmetric [20], which means any pair of opposite edges, if it exists, is identically signed $a_{i j} a_{j i}>0$. A signed digraph $\mathcal{G}^{s}=\left(\mathbb{V}^{s}, \mathbb{E}^{s}\right)$ is called a subgraph of $\mathcal{G}$ if $\mathbb{V}^{s} \subseteq \mathbb{V}$ and $\mathbb{E}^{s} \subseteq \mathbb{E}$. A signed graph is called balanced if $\sum(j, i) \in \mathbb{E}\left|a_{i j}\right|=\sum_{(i, j) \in \mathbb{E}}\left|a_{j i}\right|$ for all $i \in \mathbb{V}$. A path connecting nodes $i$ and $j$ is a sequence of distinct nodes $i_{0}:=i, i_{1}, \ldots, i_{p-1}, i_{p}:=j(k>1)$ such that $\left(i_{q-1}, i_{q}\right) \in \mathbb{E}$ for $q=1, \ldots, p$. A signed graph is quasi-strongly connected (QSC) if it has at least one node, called root, which can reach any other nodes of the graph by a path. A signed graph is strongly connected if any node can reach any other nodes of the graph with a path.

Slightly different from the conventional definition of the Laplacian matrix for unsigned graphs, the Laplacian $\mathbf{L}$ of a signed graph is denoted by

$$
\left\langle H_{i j}:= \begin{cases}-a_{i j} & \text { if } j \neq i \\ \sum_{p=1}^{n}\left|a_{i p}\right| & \text { if } j=i\end{cases}\right.
$$

According to the Gershgorin disk theorem, $\mathbf{L}$ has no eigenvalues in the closed left-half complex plane with the possible exception of eigenvalue 0 . Unlike the unsigned case, the Laplacian of graphs with negative coupling may have no eigenvalue 0 . In many opinion dynamics literature [16], [17], structural balance theory is widely used to characterize the existence of eigenvalue 0 for the signed Laplacian. A directed signed graph $\mathcal{G}$ with vertex set $\mathbb{V}$ is structurally balanced (SB) if $\mathbb{V}$ can split into two disjoint subsets (i.e., $\mathbb{V}^{+} \cup \mathbb{V}^{-}=\mathbb{V}, \mathbb{V}^{+} \cap \mathbb{V}=-\emptyset$ ) such that the weights of $(i, j) \in \mathbb{E}$ are positive $\forall i \in \mathbb{V}^{+}, j \in \mathbb{V}^{+}$and $\forall i \in \mathbb{V}^{-}, j \in \mathbb{V}^{-}$and, weights of $(i, j) \in \mathbb{E}$ are negative $\forall i \in \mathbb{V}^{+}, j \in \mathbb{V}^{-}$and $\forall j \in \mathbb{V}^{+}, i \in \mathbb{V}^{-}$. The above structural condition depicts the scenario that a group separates into two subgroups (such as advocates of two political parties), where an agent cooperates with other group-mates while competing with agents in the opposite group. The SB condition always holds in graphs without negatively weighted edges (therein one of the group is empty). As shown in [20], a quasi-strongly connected signed graph $\mathcal{G}$ that is structurally balanced implies that its Laplacian $\boldsymbol{L}$ has a simple eigenvalue at zero and all other eigenvalues have a positive real part. Finally, the associated unsigned graph of a signed graph $\mathcal{G}$ possesses the same sets of nodes and edges as $\mathcal{G}$ and its associated Laplacian $\hat{\boldsymbol{L}}$ with $[\hat{\boldsymbol{L}}]_{i j}=-\left|[\boldsymbol{L}]_{i j}\right|$ for $i \neq j$ and $[\hat{\boldsymbol{L}}]_{i j}=[\boldsymbol{L}]_{i j}$ for $i=j$.

\subsection{Port-Hamiltonian Representation and Passivity}

We follow the convention introduced in [21]. Consider an input-output dynamical system

$$
\dot{\boldsymbol{x}}(t)=\boldsymbol{f}(\boldsymbol{x})+\boldsymbol{g}(\boldsymbol{x}) \boldsymbol{u}(t), \quad \boldsymbol{y}(t)=\boldsymbol{h}(\boldsymbol{x})
$$

where $\boldsymbol{x} \in \mathbb{R}^{n^{x}}, \boldsymbol{u} \in \mathbb{R}^{n^{u}}$ and $\boldsymbol{y} \in \mathbb{R}^{n^{y}}$ are the state, control and output, respectively. Let $\boldsymbol{f}, \boldsymbol{g}$ be locally Lipschitz and $\boldsymbol{h}$ be continuous, satisfying $f(0)=\mathbf{0}, g(0)=0$ and $\boldsymbol{h}(\mathbf{0})=\mathbf{0}$. Consequently, the solution to the system (1) is unique for any locally bounded input $\boldsymbol{u}$ and the initial condition $\boldsymbol{x}(0) \in \mathbb{R}^{n^{x}}$.

The system (1) admits a port-Hamiltonian (PH) representation (in a generalized sense) if there exist $n^{x} \times n^{x}$ matrices $\boldsymbol{J}(\boldsymbol{x}), \boldsymbol{R}(\boldsymbol{x})$ satisfying $\boldsymbol{J}(\boldsymbol{x})=-\boldsymbol{J}^{\top}(\boldsymbol{x})$ and $\boldsymbol{R}(\boldsymbol{x})=\boldsymbol{R}^{\top}(\boldsymbol{x}) \succeq 0$, and a smooth function $S(\boldsymbol{x})$, called Hamiltonian, such that (1) can be rewritten in the form

$$
\begin{aligned}
\dot{\boldsymbol{x}}(t) & =(\boldsymbol{J}(\boldsymbol{x})-\boldsymbol{R}(\boldsymbol{x})) \frac{\partial S}{\partial \boldsymbol{x}}(\boldsymbol{x})+\boldsymbol{g}(\boldsymbol{x}) \boldsymbol{u}(t), \\
\boldsymbol{y} & =\boldsymbol{g}^{\boldsymbol{\top}}(x) \frac{\partial S}{\partial \boldsymbol{x}}(\boldsymbol{x}) .
\end{aligned}
$$

An appealing feature of the $\mathrm{PH}$ formulation is the passivity property when $S(\boldsymbol{x}) \geq 0$, namely

$$
\dot{S}(\boldsymbol{x})=-\frac{\partial S^{\top}}{\partial \boldsymbol{x}}(\boldsymbol{x}) \boldsymbol{R}(\boldsymbol{x}) \frac{\partial S^{\top}}{\partial \boldsymbol{x}}(\boldsymbol{x})+\frac{\partial S^{\top}}{\partial \boldsymbol{x}}(\boldsymbol{x}) \boldsymbol{g}(\boldsymbol{x}) \boldsymbol{u}(t) \leq \boldsymbol{y}(t)^{\top} \boldsymbol{u}(t) .
$$


An agent with dynamics (1) is passive if there exists a smooth function $S(\boldsymbol{x}) \geq 0$ such that the passivity inequality (3) holds. In addition, we call the passive system (1) satisfying the reformulation (2) a port-controlled Hamiltonian (PCH) system, denoted by $(\boldsymbol{J}, \boldsymbol{R}, S)$.

The $\mathrm{PH}$ approach has prominent advantages in analysis and modeling of systems from various physical domains (e.g., mechanical, electrical, electromagnetic, and thermal systems.). As one will see, the PH modeling is instrumental in our dynamical behavior analysis of opinion dynamics and information flow in social networks. Particularly, the intrinsic passive nature provides a significant implication into the convergence property of opinion systems. Much effort has been devoted to using passivity-based technique into the cooperative control of multi-agent systems with nonlinear heterogeneous dynamics [22], 23].

\subsection{Problem Formulation and Elementary Results}

Consider a network of $n \geq 2$ actors described by a signed graph $\mathcal{G}^{a}=\left(\mathbb{V}^{a}, \mathbb{E}^{a}\right)$ associated with the signed Laplacian $\boldsymbol{L}^{a} \in \mathbb{R}^{n \times n}$. Each actor $i$ has a vector $\boldsymbol{x}_{i} \in \mathbb{R}^{n^{x}}$ that represents its opinions on $n^{x}$ different subjects. In this article, we focus on continuous-valued opinions like the degree of preference to issues or the tendency to change thoughts: sign qualifies the current belief tendency -positive for support, negative for protest and zero for neutrality-, and modulus quantifies the magnitude. The time-evolution of the opinion vector $\boldsymbol{x}_{i}$ obeys the linear time-invariant (LTI) dynamics

$$
\begin{aligned}
\dot{\boldsymbol{x}}_{i}(t) & =\boldsymbol{F}_{i} \boldsymbol{x}_{i}(t)+\boldsymbol{G}_{i} \boldsymbol{u}_{i}(t), \quad \boldsymbol{y}_{i}(t)=\boldsymbol{H}_{i} \boldsymbol{x}_{i}(t) \\
\boldsymbol{u}_{i}(t) & =\sum_{j=1}^{n}\left|a_{i j}\right|\left(\boldsymbol{y}_{j}(t) \operatorname{sgn} a_{i j}-\boldsymbol{y}_{i}(t)\right),
\end{aligned}
$$

where $u_{i}, y_{i} \in \mathbb{R}^{n^{y}}$ stand for the control inputs and the outputs, respectively. The matrices $\boldsymbol{F}_{i}, \boldsymbol{G}_{i}$ and $\boldsymbol{H}_{i}$ with appropriate dimensions denote the open loop system matrix, the control input to the state map, and the state to output map, respectively. Here, the coupling weights $a_{i j} \in \mathbb{R}$, can accommodate antagonistic interactions. That is to say, there may exist edges in graph $\mathcal{G}^{a}$ with coupling weights $a_{i j}<\theta$ for $(j, i) \in \mathbb{E}^{a}$.

Remark 1. In the recent literature, extensive works have demonstrated that noise (e.g., communication uncertainty between agents and exogenous disturbance due to an external environment) can indeed dramatically affect the collective opinion formation processes. Noise, far from just a nuisance, has begun to be noticed for its essential role in social activities. In fact, injecting noise properly can drive a network of agents toward desired collective behavior patterns including opinion synchronization [24], neutralization [4], and separation [25].

We start off by providing the mathematical deseriptions of collective behaviors emerging from opinion networks with antagonism.

Definition 1. An opinion dynamic is said to establish output modulus synchronization under a prescribed control protocol, if for any initial states, the following statement

$$
\lim _{t \rightarrow \infty}\left|\boldsymbol{y}_{i}(t)\right|-\left|\boldsymbol{y}_{j}(t)\right|=\mathbf{0}, \quad \lim _{t \rightarrow \infty}\left\|\boldsymbol{y}_{i}(t)\right\|<\infty,
$$

holds for all $i, j \in \mathbb{V}^{a}$. With additional conditions, the output modulus synchronization can be further categorized:

1). if $\lim _{t \rightarrow \infty} \boldsymbol{y}_{i}(t)=\mathbf{0}$ for all $i \in \mathbb{V}^{a}$, then we say the protocol establishes output neutralization,

2). otherwise, we say the protocol establishes output bipartite synchronization. In particular,

2.1). if $\lim _{t \rightarrow \infty} \boldsymbol{y}_{i}(t)-\boldsymbol{y}_{j}(t)=\mathbf{0}$ for all $i, j \in \mathbb{V}^{a}$, then we say the protocol establishes output synchronization,

2.2). otherwise, it establishes output polarization.

Note that since we study the opinion discussion on multiple issues, namely, the opinion variable is a vector value rather than a scalar value; thus the case when some (not all) of the entries of the opinion vector are 0 is also allowed for output bipartite synchronization.

Remark 2. The aim of this article is only to investigate whether or not the deviations between modular outputs vanish, while the outputs themselves may or may not converge to a static equilibrium vector. Consequently, we use the term "modulus/bipartite synchronization" in Definition 1 by extending the relevant concepts of multi-agent cooperative control with antagonistic interaction [26], [27]. It is different from the usual modulus/bipartite consensus definition introduced in [16], [20], in which the outputs or rather states of all social actors are required to converge in modulus to a constant. Hence, the bipartite consensus of opinion dynamics implies bipartite synchronization, but not necessarily the other way around. See [3] and the references therein for more discussions on the conceptual definition of modulus consensus/synchronization/flocking. Moreover, we use the terminology "neutralization" to characterize the phenomenon where social actors increasingly become getting used to indifference no matter what their initial intentions were. As claimed in [28], it is a more descriptive term in social science than the technical term of stabilization used in Altafinis' work [16].

Throughout this article, we primarily deal with dynamical systems of the $\mathrm{PH}$ formulation. More details on the interpretation of the above model and its sociological implications will be provided later. 
TABLE 1: Glossary of Terms

\begin{tabular}{l|cccc}
\hline social network & social entity & social actor & social media & social community \\
graph theory & node & ordinary node & dominant node & subgroup \\
biological example & living organism & species & intermediate & cell \\
governance system & people & civilian & legislator & factor \\
election campaign & citizen & constituent & elected official & political parity \\
social movement & participator & common participator & radical leader & clique \\
factory, company & staff & worker, clerk & managers, administrator & office, department \\
information systems & gossiper & audience & press & cumulative audience \\
commercial market & user & customer & advertiser & user group \\
\hline
\end{tabular}

Assumption 1. Actors with dynamics (4) admit representations in the form of PCH systems $\left(\boldsymbol{J}_{i}^{a}, \boldsymbol{R}_{i}^{a}, S_{i}^{a}\right)$ :

$$
\dot{\boldsymbol{x}}_{i}(t)=\left(\boldsymbol{J}_{i}^{a}-\boldsymbol{R}_{i}^{a}\right) \frac{\partial S_{i}^{a}}{\partial \boldsymbol{x}_{i}}\left(\boldsymbol{x}_{i}\right)+\boldsymbol{G}_{i} \boldsymbol{u}_{i}, \quad \boldsymbol{y}_{i}=\boldsymbol{G}_{i}^{\top} \frac{\partial S_{i}^{a}}{\partial \boldsymbol{x}_{i}}\left(\boldsymbol{x}_{i}\right) .
$$

with a skew-symmetric matrix $\boldsymbol{J}_{i}^{a}=-\left(\boldsymbol{J}_{i}^{a}\right)^{\top}$, a positive semi-definite matrix $\boldsymbol{R}_{i}^{a}=\left(\boldsymbol{R}_{i}^{a}\right) \succeq 0$, and a radially unbounded Hamiltonian function $S_{i}^{a} \geq 0$.

For cooperative networks, it is widely known that passive agents achieve output synchronization if the interaction graph is strongly connected and balanced [22]. By giving some sufficient conditions, we first provide a criterion to establish output modulus synchronization of opinion systems $4 \mathrm{4a}$ ) with the control law $4 \mathrm{~b}$ ) on coopetitive (cooperative-competitive) networks.

Proposition 1. Consider the opinion dynamics (4) under Assumption 1 for all actors $i \in \mathbb{V}^{a}$. If the signed graph $\mathcal{G}^{a}$ is quasi-strongly connected and balanced, then the protocol (4b) establishes output modulus synchronization.

Proof. Due to the PH representation of actors, the Hamiltonian of the entire actor-to-actor network is the summation of the individual Hamiltonian, i.e., $S^{a}=\sum_{i}^{n} S_{i}^{a}$ whose time derivative along the trajectories of the system (4) satisfies

$$
\dot{S}^{a}(\boldsymbol{x})=\frac{d}{d t} \sum_{i=1}^{n} S_{i}^{a}\left(\boldsymbol{x}_{i}\right) \leq \sum_{i=1}^{n}\left(\boldsymbol{y}_{i}^{\top}(t) \boldsymbol{u}_{i}(t)\right)=\sum_{i, j=1}^{n} a_{i j} \boldsymbol{y}_{i}^{\top}(t) \boldsymbol{y}_{j}(t)-\left|a_{i j}\right| \boldsymbol{y}_{i}^{\top}(t) \boldsymbol{y}_{i}(t),
$$

where $\boldsymbol{x}=\left[\boldsymbol{x}_{1}^{\top}, \ldots, \boldsymbol{x}_{n}^{\top}\right]^{\top} \in \mathbb{R}^{n n^{x}}$. The balancedness of the digraph $\mathcal{G}^{a}$, i.e., $\sum_{j=1}^{n}\left|a_{i j}\right|=\sum_{j=1}^{n}\left|a_{j i}\right|$ implies that the additive power is further equal to

$$
\begin{aligned}
\sum_{i=1}^{n} \boldsymbol{y}_{i}^{\top}(t) \boldsymbol{u}_{i}(t) & =\sum_{i, j=1}^{n}\left(a_{i j} \boldsymbol{y}_{i}^{\top}(t) \boldsymbol{y}_{j}(t)-\frac{1}{2}\left|a_{i j}\right|\left(\left\|\boldsymbol{y}_{i}(t)\right\|^{2}+\left\|\boldsymbol{y}_{j}(t)\right\|^{2}\right)\right) \\
& =-\frac{1}{2} \sum_{i, j}^{n}\left|a_{i j}\right|\left\|\boldsymbol{y}_{j}(t) \operatorname{sgn} a_{i j}-\boldsymbol{y}_{i}(t)\right\|^{2} \leq 0
\end{aligned}
$$

Since $S^{a}$ is proper due to the local positive definiteness and the radial unboundedness, the inequality (6) along with (5) implies that the solution $\boldsymbol{x}(t)$ to the systems $(4)$ is bounded and stays in a compact set. The fact that $\boldsymbol{H}_{i}$ is a real matrix for $i \in \mathbb{V}^{a}$ indicates the output signals $\boldsymbol{y}(t)$ are also bounded, i.e., $\lim _{t \rightarrow \infty}\|\boldsymbol{y}(t)\|<\infty$. By the application of LaSalle invariant principle and the fact sgn $a_{i j} \in\{ \pm 1\}, \boldsymbol{x}(t)$ converges to a $\omega$-limit set in which $\left|\boldsymbol{y}_{i}(t)\right|=\left|\boldsymbol{y}_{j}(t)\right|$ for all $\hat{\imath}_{;} j \in \mathbb{V}^{a}$. It is known that a balanced graph is quasi-strongly connected if and only if it is strongly connected. Following by Theorem 8.5 of [22], for arbitrary initial states $\boldsymbol{x}(0) \in \mathbb{R}^{n n^{x}}$, the system (5) reaches output modulus synchronization, i.e.

$$
\lim _{t \rightarrow \infty}\left|\boldsymbol{y}_{i}(t)\right|-\left|\boldsymbol{y}_{j}(t)\right|=\mathbf{0}, \quad \forall i, j \in \mathbb{V}^{a} .
$$

The proof of Proposition 1 is complete.

In the real world, however, people are more or less exposed to pervasive mass and electronic media in their social surrounding. Hence, a natural question is how the opinion of actors evolves under the influence of social media. To this end, we first modify the model of opinion dynamics (4) in the following way.

Consider the existence of $m>1$ media in a social network. Let $\mathcal{G}^{m}=\left(\mathbb{V}^{m}, \mathbb{E}^{m}\right)$ be a graph describing the underlying interconnection structure among media with $\mathbb{V}^{m}=\{1, \ldots, m\}$ and $\mathbb{E}^{m} \subseteq \mathbb{V}^{m} \times \mathbb{V}^{m}$. To capture market segmentation in media industries, the appearance of $m$ media recasts the actor-to-actor communication network $\mathcal{G}^{a}$ consisting of $m$ subgraphs: Let $\mathcal{G}_{k}^{a}=\left(\mathbb{V}_{k}^{a}, \mathbb{E}_{k}^{a}\right)$ be the subgraph characterizing the interconnection relation among actors confronting medium $k \in \mathbb{V}^{m}$, where $\left|\mathbb{V}_{k}^{a}\right|=n_{k}$ and $(i, j) \in \mathbb{E}_{k}^{a}$ if $(i, j) \in \mathbb{E}^{a}$ and $i, j \in \mathbb{V}_{k}^{a}$. For convenience, we label the first $n_{1}$ nodes of the graph as the actors exposed to medium 1 and so on. Therefore, one has $n_{1}+\cdots+n_{m}=n, \mathbb{V}_{1}^{a} \cup \cdots \cup \mathbb{V}_{m}^{a}=\mathbb{V}_{a}$, 
and $\mathbb{E}_{1}^{a} \cup \cdots \cup \mathbb{E}_{m}^{a}=\mathbb{E}^{a}$. As such, media influence and community-based architecture render the actors the following opinion update rule

$$
\begin{aligned}
& \dot{\boldsymbol{x}}_{i}(t)=\boldsymbol{F}_{\gamma(i)} \boldsymbol{x}_{i}(t)+\boldsymbol{G}_{\gamma(i)} \boldsymbol{u}_{i}(t), \quad \boldsymbol{y}_{i}(t)=\boldsymbol{H}_{\gamma(i)} \boldsymbol{x}_{i}(t), \\
& \boldsymbol{u}_{i}(t)=\sum_{j \in \mathbb{V}_{\gamma(i)}^{a}} a_{i j}\left(\boldsymbol{y}_{j}(t)-\boldsymbol{y}_{i}(t)\right)+b_{i \gamma(i)}\left(\boldsymbol{\eta}_{\gamma(i)}(t)-\boldsymbol{y}_{i}(t)\right),
\end{aligned}
$$

where $a_{i j} \in \mathbb{R}_{>0}$ and $b_{i k} \in \mathbb{R}_{>0}$ stand for the degree of confidence or susceptibility to interactions. $\gamma: \mathbb{V}^{a} \rightarrow \mathbb{V}^{m}$ is a surjective function defined between two sets of indices (not necessarily injective). Two actors $i, j \in \mathbb{V}^{a}$ are said to be in the same group iff $\gamma(i)=\gamma(j)$. In the controller update rule (??), $\boldsymbol{\eta}_{\gamma(i)}$ is the output of medium $\gamma(i) \in \mathbb{V}^{m}$ whose dynamical evolution obeys the following update rule

$$
\begin{aligned}
& \dot{\boldsymbol{\chi}}_{k}(t)=\boldsymbol{\Psi}_{k} \boldsymbol{\chi}_{k}(t)+\boldsymbol{\Theta}_{k} \boldsymbol{\mu}_{k}(t), \quad \boldsymbol{\eta}_{k}(t)=\boldsymbol{\Xi}_{k} \boldsymbol{\chi}_{k}(t), \\
& \boldsymbol{\mu}_{k}(t)=\sum_{l \in \mathbb{V}^{m}}\left|c_{k l}\right|\left(\boldsymbol{\eta}_{l}(t) \operatorname{sgn} c_{k l}-\boldsymbol{\eta}_{k}(t)\right)+\sum_{j \in \mathbb{V}_{k}^{a}} d_{k j}\left(\boldsymbol{y}_{j}(t)-\boldsymbol{\eta}_{k}(t)\right),
\end{aligned}
$$

where $\boldsymbol{\chi}_{k} \in \mathbb{R}^{n^{x}}$ and $\boldsymbol{\mu}_{k}, \boldsymbol{\eta}_{k} \in \mathbb{R}^{n^{y}}$ respectively are state, input, and output of medium $k$. In eq. $8 \mathrm{~b}$,,$c_{k l} \in \mathbb{R}$ and $d_{k j} \in \mathbb{R}_{>0}$ are coupling strength of the interaction from media and actors, respectively. In comparison to the previous actor-to-actor network dynamics (4), we assume no antagonism in interpersonal communication to avoid unnecessary complexity, i.e., $a_{i j} \geq 0$, whereas the interaction coefficients $c_{k l}$ allow negative values, making possible the representation of competition relationship among media. Qualitatively, the development of the article can also be adapted to the negative ties among actors.

The compartmental model (7) of opinion dynamics resembles the cell-based architecture in biological systems [29| as shown in Figure 1. and is indeed ultimately related to the emergence and prevalence of clustering phenomenon among reallife groups typically consisting of like-minded individuals [30]. For example, homophily (media are "heard" only by persons with views being not too far away) provides the sociological explanation for the appearance of social agglomerations. Moreover, such social network models are reminiscent of many paradigms in the biological world and human society; see Table 11, but we will not expand further. Compared with simple actor-to-actor networks, the quorum-sensing interaction structure is, in some sense, far more robust against local disturbance as media can somehow "filter" out noise [4].

Remark 3. Notably, the developed results in this article are independent of the specific community-detection algorithm, especially of topological operations such as zoom in or out of compartments, which do not change the collective opinion evolution in social spaces. The topological structure in which each group $\mathcal{G}_{k}^{a}$ connects to one medium $k$ does not represent a restriction and applies to a more general setup wherein the opinions of media available for an actor group are in the same phase. Throughout the article, we only focus on the model 7 for simplicity of analysis and clarity of presentation.

In what follows, we study in depth the evolutionary behavior of individual opinions under the joint influence of the continuous communication with neighboring peers and the assimilation of information advocated on media.

\section{Main Results}

This section provides some fundamental properties and convergence property for the proposed mathematical model describing opinion dynamics.

\subsection{Convergence Behavior Analysis}

Before embarking on the detailed analysis, we first introduce some notational conventions and topological properties of the graph modeling the social networks.

As a consequence of media description in (8), the signed Laplacian matrix $\boldsymbol{L}^{m} \in \mathbb{R}^{m \times m}$ associated to graph $\mathcal{G}^{m}$ is given by $\left[\boldsymbol{L}^{m}\right]_{k l}:=-c_{k l}$ if $k \neq l$ and $\sum_{p=1}^{m}\left|c_{k p}\right|$ if $k=l$. Let $\boldsymbol{L}_{k}^{a} \in \mathbb{R}_{>0}^{n_{k} \times n_{k}}$ be the Laplacian matrix of subgraph $\mathcal{G}_{k}^{a}$ for $k \in\{1, \ldots, m\}$, so that $\boldsymbol{L}^{a}=\operatorname{diag}\left(\boldsymbol{L}_{1}^{a}, \ldots, \boldsymbol{L}_{m}^{a}\right)$. Therefore, we can formalize the interaction structure encoded in the control protocol (??) and $8 \mathrm{~b}$ by an $(n+m) \times(n+m)$ Laplacian matrix $\boldsymbol{L}^{e} \triangleq\left[\begin{array}{cc}\boldsymbol{L}^{a}+\boldsymbol{B} & -\tilde{\boldsymbol{B}} \\ -\tilde{\boldsymbol{D}} & \boldsymbol{L}^{m}+\boldsymbol{D}\end{array}\right]$ where

$$
\begin{aligned}
& \tilde{\boldsymbol{B}} \in \mathbb{R}_{\geq 0}^{n \times m}=\operatorname{diag}\left(\boldsymbol{b}_{1}, \ldots, \boldsymbol{b}_{m}\right), \quad \text { with } \boldsymbol{b}_{k}=\left[b_{\left(\sum_{l=1}^{k-1} n_{l}+1\right) k}, \ldots, b_{\left(\sum_{l=1}^{k} n_{l}\right) k}\right]^{\top} \\
& \boldsymbol{B} \in \mathbb{R}_{\geq 0}^{n \times n}=\operatorname{diag}\left(\boldsymbol{B}_{1}, \ldots, \boldsymbol{B}_{m}\right), \quad \text { with } \boldsymbol{B}_{k}=\operatorname{diag}\left(b_{\left(\sum_{l=1}^{k-1} n_{l}+1\right) k}, \ldots, b_{\left(\sum_{l=1}^{k} n_{l}\right) k}\right), \\
& \tilde{\boldsymbol{D}} \in \mathbb{R}_{\geq 0}^{m \times n}=\operatorname{diag}\left(\boldsymbol{d}_{1}, \ldots, \boldsymbol{d}_{m}\right), \quad \text { with } \boldsymbol{d}_{k}=\left[d_{k\left(\sum_{l=1}^{k-1} n_{l}+1\right)}, \ldots, d_{k\left(\sum_{l=1}^{k} n_{l}\right)}\right] \\
& \boldsymbol{D} \in \mathbb{R}_{\geq 0}^{m \times m}=\operatorname{diag}\left(\sum_{j \in \mathbb{V}_{1}^{a}} d_{1 j}, \ldots, \sum_{j \in \mathbb{V}_{m}^{a}} d_{m j}\right) .
\end{aligned}
$$


This Laplacian matrix $\boldsymbol{L}^{e}$ designates an adjacency matrix $\boldsymbol{W}=\left[w_{i j}\right] \in \mathbb{R}^{(n+m) \times(n+m)}$ with the elements $w_{p q}=-[\boldsymbol{L}]_{p q}$ and $w_{p p}=0$ for $p, q=1, \ldots, n+m$. In what follows, we use graph $\mathcal{G}^{e}=\left(\mathbb{V}^{e}, \mathbb{E}^{e}\right)$ to represent the underpinning interconnection structure of the entire social network consisting of actors and media, wherein $\mathbb{V}^{e}=\{1, \ldots, n, n+1, \ldots, n+m\}$ is vertex set and the edge set $\mathbb{E}^{e} \subseteq \mathbb{V}^{e} \times \mathbb{V}^{e}$ has elements $(q, p) \in \mathbb{E}^{e}$ if $w_{p q} \neq 0$ and $q \neq p$. Without loss of generality, the media are labeled by $\{n+1, \ldots, n+m\}$ in graph $\mathcal{G}^{e}$.

Similar to the treatment of actor systems, we restrict ourselves to the passive media dynamics satisfying the following assumptions.

Assumption 2. For all $k \in \mathbb{V}^{m}$, medium $k$ with dynamics (8) is of the PH representation (2) associated with a skewsymmetric matrix $\boldsymbol{J}_{k}^{m}=-\left(\boldsymbol{J}_{k}^{m}\right)^{\top}$, a positive semi-definite matrix $\boldsymbol{R}_{k}^{m}=\left(\boldsymbol{R}_{k}^{m}\right)^{\top} \succeq 0$, and a radially unbounded Hamiltonian $S_{k}^{m} \geq 0$.

Assumption 3. In each actor subgroup, at least one actor is listening to the medium, i.e., $\sum_{i \in \mathbb{V}_{k}^{a}} b_{i k}>0$ for all $k \in \mathbb{V}^{m}$ and the actor-media interaction is equivalently reciprocal, i.e., $b_{i k}=d_{k i}$ for all $i \in \mathbb{V}^{a}$ and $k \in \mathbb{V}^{m}$.

Note that Assumption 3 is necessary to derive the primary results in this article. The following Lemma exposes some elementary topological properties of graph $\mathcal{G}^{e}$.

Lemma 1. For a social network with $n$ actors and $m$ media,

1). graph $\mathcal{G}^{e}$ is structurally balanced if and only if media graph $\mathcal{G}^{m}$ is structurally balanced.

Furthermore, when Assumption 3 holds,

2). if media graph $\mathcal{G}^{m}$ and all actor subgraphs $\mathcal{G}_{k}^{a}\left(k \in \mathbb{V}^{m}\right)$ are respectively balanced, then graph $\mathcal{G}^{e}$ is balanced;

3). if graph $\mathcal{G}^{m}$ is quasi-strongly connected and all subgraphs $\mathcal{G}_{k}^{a}(k=1, \ldots, m)$ are strongly connected, then

3.1). $\quad$ graph $\mathcal{G}^{e}$ is quasi-strongly connected;

3.2). there is at least one dominant node (medium) being a root of graph $\mathcal{G}^{e}$;

3.3). there is at least one ordinary node (actor) being a root of graph $\mathcal{G}^{e}$.

Proof. Since each group of actors only listens to one specific medium and the edges with negative weights only appear in the interactions among media, one direction of claim 1). is obvious: if $\mathcal{G}^{e}$ is structurally balanced, media graph $\mathcal{G}^{m}$ is structurally balanced. Similarly, if $\mathcal{G}^{m}$ is structurally balanced, then $\mathbb{V}^{m}$ can be split into two hostile camps and nodes in $\mathbb{V}^{a}$ can be allocated at any one of them, leading to the structural balance of graph.

Suppose that the media graph is quasi-strongly connected, let medium $1 \in \mathbb{V}^{m}$ (equivalently, node $n+1 \in \mathbb{V}$ in graph $\left.\mathcal{G}^{e}\right)$ be the root of graph $\mathcal{G}^{m}$, which has at least one path to connect to any other media. Since Assumption 3 is satisfied, the strong connectedness of each actor subgraph $\mathcal{G}_{k}^{a}$ implies each medium $k$ in $\mathbb{V}^{m}$ has a path to communicate with all nodes in $V_{k}^{a}$. Consequently, one can always find a path connecting from media 1 to all actors and thus condition 3.2) holds. The implication 3.1). follows straightforwardly from condition 3.3).

Moreover, there exists at least one actor in $\mathbb{V}_{1}^{a}$, supposed the node with label $1 \in \mathbb{V}^{a}$ (equivalently, node $1 \in \mathbb{V}$ in graph $\left.\mathcal{G}^{e}\right)$, satisfying $d_{(n+1) 1}=b_{1(n+1)}>0$ according to Assumption 3 Namely, both actor 1 and medium $n+1$ on graph $\mathcal{G}^{e}$ have a link connecting to another. The fact that the media node $1 \in \mathbb{V}^{m}$ is a root of graph $\mathcal{G}^{e}$ implies the actor $1 \in \mathbb{V}^{a}$ has a path on graph $\mathcal{G}^{e}$ to reach other nodes in $\mathbb{V}^{e}$ by transiting though medium $n+1 \in \mathbb{V}$. Therefore, actor 1 is also a root of graph $\mathcal{G}^{e}$ and the proof is finished.

In analogy with Proposition 1 in simple actor-to-actor communication structure, we can provide a criterion for output modulus synchronization over the network $\mathcal{G}^{e}$.

Theorem 1. Consider $n$ actors of dynamics (7) under Assumption 11 and m media of dynamics (8) under Assumption 2 If the following topological conditions hold:

1). Assumption 3 holds;

2). subgraphs $\mathcal{G}_{k}^{a}$ are balanced and strongly connected for all $k \in \mathbb{V}^{m}$;

3). subgraph $\mathcal{G}^{m}$ is balanced and quasi-strongly connected;

then all actors (7) reach output modulus synchronization.

Proof. Obviously, graph $\mathcal{G}^{e}$ is quasi-strongly connected and balanced as a result of Lemma 1

Let the Hamiltonian of the entire social network be

$$
S(\boldsymbol{x}, \boldsymbol{\chi})=\sum_{i=1}^{n} S_{i}^{a}\left(\boldsymbol{x}_{i}\right)+\sum_{k=1}^{m} S_{k}^{m}\left(\boldsymbol{\chi}_{k}\right)
$$

where $\boldsymbol{x}=\left[\boldsymbol{x}_{1}^{\top}, \ldots, \boldsymbol{x}_{n}^{\top}\right]^{\top}$ and $\left[\boldsymbol{\chi}_{1}^{\top}, \ldots, \boldsymbol{\chi}_{m}^{\top}\right]^{\top}$ are the stacked vectors of actors and media, respectively. By treating media as members of the actor-to-actor network, Proposition 1 with the Hamiltonian function 9 9 implies the protocol (??) synchronizes the outputs of systems $7 \mathrm{7a}$ in modulus for every choice of the initial conditions, i.e.,

$$
\lim _{t \rightarrow \infty}\left|\boldsymbol{y}_{i}(t)\right|-\left|\boldsymbol{y}_{j}(t)\right|=\mathbf{0}, \quad \forall i, j \in \mathbb{V}^{a},
$$


for all initial conditions $\boldsymbol{x}(0) \in \mathbb{R}^{n n^{x}}, \chi(0) \in \mathbb{R}^{m n^{x}}$. Thus, the proof is complete.

Although not explicit in Theorem 1 , the media $8 \mathrm{a}$ ) also establishes output modulus synchronization under the control rule (8b), i.e., $\lim _{t \rightarrow \infty}\left|\boldsymbol{\eta}_{k}(t)\right|-\left|\boldsymbol{\eta}_{l}(t)\right|=\mathbf{0}$, for all $k, l \in \mathbb{V}^{m}$, independently of the initial conditions. More significantly, one can also check that $\lim _{t \rightarrow \infty}\left|\boldsymbol{y}_{i}(t)\right|-\left|\boldsymbol{\eta}_{k}(t)\right|=\mathbf{0}$ for all $i \in \mathbb{V}^{a}$ and $k \in \mathbb{V}^{m}$. That is to say, all of the social entities in the social network $\mathcal{G}^{e}$ asymptotically reach output modulus synchronization at the end of the issue discussion.

In cooperative networks [22], no explicit extraction of the trivial case that the synchronous trajectories asymptotically vanish independent of initial states is made on the notion of synchronization. Yet, it is important to highlight such distinction of synchronous behavior in opinion dynamics, since the "degenerate" case implies all of the opinions become neutralized in the end and is of essential interest in its own right.

Before getting into the details, we define an equivalence relation $\sim_{s}$ on system matrices $\boldsymbol{F}_{i}$ and $\boldsymbol{F}_{j}$ by $\boldsymbol{F}_{i} \sim_{s} \boldsymbol{F}_{j}$ if they satisfy the properties

1). $\operatorname{sp}\left(\boldsymbol{F}_{p}\right) \cap \iota \mathbb{R} \neq \emptyset(p=i, j)$ and those eigenvalues are semi-simple;

2). $\operatorname{sp}\left(\boldsymbol{F}_{i}\right) \cap \iota \mathbb{R}=\operatorname{sp}\left(\boldsymbol{F}_{j}\right) \cap \iota \mathbb{R} ;$

3). the eigen-spaces corresponding respectively to $\operatorname{sp}\left(\boldsymbol{F}_{i}\right) \cap \iota \mathbb{R}$ and $\operatorname{sp}\left(\boldsymbol{F}_{j}\right) \cap \iota \mathbb{R}$ are the same.

Note that the definition of this equivalence relation implies the matrix $F_{p}(p=i, j)$ has at least one eigenvalue 0 or a pair of purely imaginary eigenvalues whose geometric multiplicity is equal to their algebraic multiplicity. In particular, those eigenvalues on the imaginary axis and the associated eigenspace are the same for all elements in the equivalence class $\left[\boldsymbol{F}_{i}\right]_{\sim_{s}}$.

Then, the following theorem serves as the investigation of the non-trivial case where output modulus synchronization implies output synchronization or output polarization.

Theorem 2. Let the assumptions and conditions of Theorem 1 hold. If the following conditions are satisfied:

1). media graph $\mathcal{G}^{m}$ is structurally balanced;

2). $\quad \boldsymbol{F}_{i} \sim_{s} \boldsymbol{F}_{j} \sim_{s} \boldsymbol{\Psi}_{k}$ for all $i, j \in \mathbb{V}^{a}$ and $k \in \mathbb{V}^{m}$;

3). $\quad \boldsymbol{H}_{i}^{\top} \boldsymbol{H}_{i} \succ 0$ for all $i \in \mathbb{V}^{a}$;

then the actor dynamics (7) reaches output bipartite synchronization for any non-zero initial conditions: if $c_{k l} \geq 0$ for all $k, l \in \mathbb{V}^{m}$, then the synchronization of output opinion is reached; otherwise, output opinions polarize.

Proof. As graph $\mathcal{G}^{e}=\left(\mathbb{V}^{e}, \mathbb{E}^{e}\right)$ is structurally balanced thanks to statement 1$)$ of Lemma 1 . we may denote $\mathbb{V}^{e-}$ and $\mathbb{V}^{e+}$ as two "antagonistic camps" such that $\mathbb{V}^{e-} \cap \mathbb{V}^{e+}=\emptyset$ and $\mathbb{V}^{e}-\mathbb{V}^{e}+\mathbb{V}^{e}$. Without loss of generality, let $\pi_{p}=1$ if $p \in \mathbb{V}^{e+}$ and $\pi_{p}=-1$ if $p \in \mathbb{V}^{e-}$. After denoting the augmented variable

$$
\begin{aligned}
& \boldsymbol{z}:=\left[\boldsymbol{z}_{1}^{\top}, \ldots, \boldsymbol{z}_{n+m}^{\top}\right]^{\top}=\left[\boldsymbol{y}^{\top}, \boldsymbol{\eta}^{\top}\right]^{\top}, \\
& \text { (9) becomes }
\end{aligned}
$$

the time-derivative of the Hamiltonian $S$ in $(9)$ becomes

$$
\begin{aligned}
\dot{S} & \leq \sum_{p, q}^{n+m}\left(w_{p q} \boldsymbol{z}_{p}^{\top} \boldsymbol{z}_{q}-\frac{1}{2}\left|w_{p q}\right|\left(\boldsymbol{z}_{p}^{\top} \boldsymbol{z}_{p}+\boldsymbol{z}_{q}^{\top} \boldsymbol{z}_{q}\right)\right) \\
& =-\frac{1}{2} \sum_{p, q}^{n+m}\left|w_{p q}\right|\left\|\boldsymbol{z}_{q} \operatorname{sgn} w_{p q}-\boldsymbol{z}_{p}\right\|^{2}
\end{aligned}
$$$$
=-\frac{1}{2} \sum_{p, q}^{n+m}\left|w_{p q}\right|\left\|\pi_{q} \boldsymbol{z}_{q}-\pi_{p} \boldsymbol{z}_{p}\right\|^{2}
$$

where the inequality (10) is similar in the spirit to (5) and the last equation (12) comes from the fact sgn $w_{p q}=\pi_{p} \pi_{q}$ for $(q, p) \in \mathbb{E}^{e}$ as graph $\mathcal{G}^{e}$ is structurally balanced.

According to LaSalle invariance principle, $\left[\boldsymbol{x}^{\top}, \boldsymbol{\chi}^{\top}\right]$ thus converges to the $\omega$-limit set $\Omega(\boldsymbol{x}(0), \chi(0))$ in which one has $\pi_{q} \boldsymbol{z}_{q}=\pi_{p} \boldsymbol{z}_{p}$ for all $p, q \in \mathbb{V}^{e}$. On this controlled invariant subspace $\Omega(\boldsymbol{x}(0), \boldsymbol{\chi}(0))$, the dynamics of actors $(7 \mathrm{a})$ reduce to an unforced system $\dot{\boldsymbol{x}}_{i}(t)=\boldsymbol{F}_{\gamma(i)} \boldsymbol{x}_{i}(t)$. Moreover, the zero vector is not an asymptotic equilibrium of this autonomous system since $\operatorname{sp}\left(\boldsymbol{F}_{\gamma}(i)\right)$ contains at least one simply stable eigenvalue according to condition 2) except the zero initial condition. The equivalence relation among all matrices $\operatorname{sp}\left(\boldsymbol{F}_{i}\right)\left(i \in \mathbb{V}^{a}\right)$ guarantees that the unforced systems perform the same longterm dynamical behavior. Thus, any actor $i \in \mathbb{V}^{a}$ must obey $\lim _{t \rightarrow \infty}\left|\boldsymbol{y}_{i}(t)\right| \neq \mathbf{0}$ due to $\boldsymbol{H}_{i} \boldsymbol{x}_{i} \neq \mathbf{0}$ for all $\boldsymbol{x}_{i} \in \mathbb{R}^{n^{x}} /\{\mathbf{0}\}$. Combining with Theorem 1. one can conclude that the actor-to-actor network reaches polarization of output opinions if there exists at least one negatively weighted edge in graph $\mathcal{G}^{m}$; otherwise, output synchronization occurs. The proof is complete.

Remark 4. It is already known that the structural balance condition endows the network topology with symmetries and drives network dynamics towards a desired synchronization or polarization pattern [16]. In addition to symmetries of network topology, the authors of [31] have shown that the symmetries of the nodes' vector fields are also of key importance to attain the specific synchronization pattern in opinion dynamics with the generalized ODE model (1). The rationale behind 
the idea is to examine $\Gamma$-equivariance of the intrinsic node dynamics. Specifically, there exist or not orthogonal matrices that commute with system matrices [32|. For structurally balanced networks, the opinion dynamics of LTI systems (4) is naturally $\Gamma$-equivariant thanks to the fact that $\pi_{i} \boldsymbol{I}$ commutes with every system matrix $\boldsymbol{F}_{i}$ where $i \in \mathbb{V}^{a}$. For more results on symmetries and equivariance in collective behavior analysis of networked systems, readers are referred to [31], [32].

Remark 5. It is worthy to note that when the system matrices $\boldsymbol{F}_{i}$ for all $i \in \mathbb{V}^{a}$ have no purely imaginary eigenvalue except one or multiple semi-simple zero eigenvalues satisfying condition 2) of Theorem 2 , the opinion-evolving process collapses to the stationary equilibrium setting, similar to the study in [16], [20]. That is, all opinion states converge asymptotically to a constant vector in a cooperative context or two opposing constant vectors when antagonism occurs. Otherwise, some components of the opinion trajectories steered by dynamics (7a) exhibit a simple harmonic oscillation as time progresses. The phenomenon that the opinion-forming process either collapses to an equilibrium state or shows permanent oscillation is related to bifurcation of dynamical systems [33]. For simplicity of presentation, we focus on the agent dynamics with the uniform system matrix $\boldsymbol{F}$ for all agents $p \in \mathbb{V}^{e}$ as an illustrative example. With the emergence of output bipartite synchronization, we present the computation of the bifurcation value for a family of system matrices. According to Theorem 2, the system matrix $\boldsymbol{F}$ in the homogeneous case is required to have at least one semi-simple eigenvalue with the zero real part. The number of these eigenvalues which are nonzero but purely imaginary plays, to some extent, the role of a bifurcation parameter and the bifurcation occurs when the number becomes zero. If the value is equal to zero, the asymptotic behavior of opinion dynamics falls into a steady-state consensus; otherwise, the system exhibits oscillatory behavior. Our statement of bifurcation phenomenon can also be generalized to a setting with heterogeneous agents. For more discussions on bifurcation analysis in multi-agent systems, see [33], [34].

According to Theorem 2, the output bipartite synchronization of opinion evolution is determined by two factors. On the one hand, the constraint is on the communication structure among participating entities. In this work, the graph-theoretic conditions (strong connectedness and balancedness) for asymptotic synchronization of passive multi-agent systems [22] are customized distributively to subgraphs of social networks. Besides, the structurally balanced condition is a significant source of the opinion cleavage in society. Specifically, the network of media with different memes might split into two disjoint camps such that media in the same club mimic the memes with each other while media coming from distinct groups confront each other for some reasons, e.g., grabbing higher audience ratings. On the other hand, the dynamic characteristics of agents are in close relation with the (nontrivial) synchronous behaviors of opinions in a population. The basic requirement on their dynamic intersection is necessary to achieve bipartite output synchronization for issue discussions. Indeed, everyday experience suggests that individuals who may be unmatched in many aspects, e.g., age, height, color, etc., should have some commonalities such as the moral compass of society, in the hope of participating collective group behaviors. In mathematics, it is of importance to strike a bargain of the spectral intersection of system matrices on complex axis.

Evidently, it is not challenging to produce a counterpart of Theorem 2 in actor-to-actor interaction networks.

Corollary 1. Consider $n$ actors of system dynamics (4) under Assumption 1 and the simple actor-to-actor network $\mathcal{G}^{a}$ being quasistrongly connected and balanced. If the following conditions are satisfied:

1). graph $\mathcal{G}^{a}$ is structurally balanced;

2). $\quad \boldsymbol{F}_{i} \sim_{s} \boldsymbol{F}_{j}$ for $i, j \in \mathbb{V}^{a}$;

3). $\quad \boldsymbol{H}_{i}^{\top} \boldsymbol{H}_{i} \succ 0$ for all $i \in \mathbb{V}^{a}$;

then actor systems (4) achieve output bipartite synchronization: if $a_{i j} \geq 0$ for all $i, j \in \mathbb{V}^{a}$, then output opinions become synchronization; otherwise, output opinions polarize.

Since the structural balance and imbalance of graphs are two mutually exclusive properties, a criterion concluding output neutralization in a coopetitive organization can be deduced from Theorem 1 and The

Theorem 3. Let the conditions of Theorem 1 hold. If the media graph is strongly connected and structurally unbalanced, then actors (7a) reach output neutralization under protocol (??) for all initial condition $\boldsymbol{x}(0) \in \mathbb{R}^{n n^{x}}$, i.e.,

$$
\lim _{t \rightarrow \infty} \boldsymbol{y}_{i}(t)=\mathbf{0}, \quad \forall i \in \mathbb{V}^{a} .
$$

In addition, if the system (7a) is zero-state detectable, then the actors attain state neutralization for all initial condition.

Proof. Inequality 11 in the proof of Theorem 2 implies that

$$
\dot{S}(\boldsymbol{x}, \boldsymbol{\chi}) \leq-\boldsymbol{z}^{\top}(t) \boldsymbol{L}^{e} \boldsymbol{z}(t) \leq-\varepsilon \sum_{p=1}^{n+m}\left\|\boldsymbol{z}_{p}(t)\right\|^{2}, \exists \varepsilon>0
$$

where the balanced condition of graph $\mathcal{G}^{e}$ is used due to Lemma 1 i.e., $\sum_{q}^{n+m} w_{p q}=\sum_{q}^{n+m} w_{q p}$ for all $p \in \mathbb{V}^{e}$. Moreover, inequality (13) also follows from Corollary 3 of [16]; in other words, if the strongly connected signed graph $\mathcal{G}^{e}$ is structural unbalance, then all of the eigenvalues of Laplacian matrix $\boldsymbol{L}^{e}$ have positive real parts.

The application of LaSalle invariance principle to the inequality (13) shows that $\left[\boldsymbol{x}^{\top}, \boldsymbol{\chi}^{\top}\right]^{\top}$ converges to the $\omega$-limit set $\Omega(\boldsymbol{x}(0), \boldsymbol{\chi}(0))$ in which one can deduce $\boldsymbol{z}_{p}(t) \rightarrow \mathbf{0}$. That is, the social network is asymptotically neutral for arbitrary initial conditions, much fewer actors, i.e., $\lim _{t \rightarrow \infty} \boldsymbol{y}_{i}(t)=\mathbf{0}$ whatever the initial conditions are. 
Furthermore, the zero-state detectability of systems leads to the reasoning that if $\boldsymbol{u}_{i} \equiv \mathbf{0}$ and $\boldsymbol{y}_{i}=\mathbf{0}$ in the $\omega$-limit set $\Omega(\boldsymbol{x}(0), \boldsymbol{\chi}(0))$, one has $\lim _{t \rightarrow \infty} \boldsymbol{x}_{i}(t)=\mathbf{0}$ for all $i \in \mathbb{V}^{a}$.

Remark 6. So far, the quest for a global criterion to identify bipartite synchronization from modulus context is an open problem for more general nonlinear systems. Although the nonlinear case is beyond the scope of this work, it is worth mentioning that by linearizing of the nonlinear dynamics in the vicinity of the original and by using Theorem (2) rooted in the linear case, one may explore some local criteria as a stepping stone.

Before closing this subsection, we provide some necessary remarks on the modeling methodology in this article. The mathematical model (7) describing opinion dynamics more generally contributes to the rapidly growing sociophysical literature [35. Indeed, the major focus in sociophysics concerns three phenomena: the compromising process (the internal interactions between actors), the effects of external influence (e.g., propaganda through media), and the self-thinking of individuals (actors are likely to change of their opinions spontaneously). Beyond that, the output-feedback mechanism in the control law design rather than state concern features the discrepancies in the private and expressed opinions of individuals on given topics. In our daily life, individuals may perform preference falsification of their real veiws for many reasons, e.g., social pressure or political future. We refer the reader to [36], [37] for empirical observations of the inconsistency between inner self-attitude and expressed opinions.

Furthermore, the adoption of the port-Hamiltonian representation to identify a prominent class of opinion dynamics provides substantial benefits in the mathematical analysis due to the natural passivity. In particular, the developed methodology allows for the interpretation of internalization, a concept studied in social psychology and sociology [18], from system and control theory. The first message is that the PH picture captures how the message (information) flow derived from the external communication with other peers and the exposure to media gets through each social actor. The passivity inequality $\dot{S}_{i}^{a}\left(x_{i}\right) \leq \boldsymbol{y}_{i}^{\top}(t) \boldsymbol{u}_{i}(t)$ is consistent with the common intuition that the abandonment rate of incoming knowledge or ideas never excesses information potential available for actors. What's more, the PH modeling of social actors illustrates that the vector field generating an opinion flow inside individuals involves a conservative and a dissipative component. Among them, the dissipative system concerning a non-negative definite matrix $\boldsymbol{R}_{i}^{a}$ governs the convergence performance of opinion dynamics, since the opinion Hamiltonian (Lyapunov-like function) decreases (gradient-descent) along the dissipative vector field. Instead, the conservative system associated with the skew-symmetric matrix $\boldsymbol{J}_{i}^{a}$ determines the equilibrium behavior after converging, agreeing with the spectral feature of the self-dynamics as revealing in Theorem 2 and Corollary 1 .

\subsection{Autocratic Media Controlling Opinion Discussions}

In the system of governance, one pitfall of the representative democracy mechanism is that elected officials are in no need to fulfill promises made before being elected, and even promote their self-interests once elected. Besides, information broadcasters like, e.g., television, newspapers, celebrity blogs, are supposed to propagate informative messages to their audiences, while they may mislead the public attitudes towards political or social events by using deliberate manipulations or even lies [38].

Those undesirable instances suggest the appearance of colluding sub-groups in social networks. More formally, they fall into a broad topic, what the sociologists call "corruption" meaning the misuse of authorized power (by heritage, education, marriage, election, appointment or whatever else) to acquire private benefits or reach ulterior purposes, e.g., in politics, economics, and culture [39]. If corruption happens in the minority, its damages include the loss of democracy, deprivation of liberty, demagoguery of public opinions, etc., necessitate the rigorous examination of the interaction organization and intrinsic mechanism in those scenarios.

First, we endow a mathematical framework to corruption in social networks, whereby one has $\sum_{i=1}^{n} \sum_{k=1}^{m} d_{i k}=0$ which means no medium is affected by actors in an autocratic society. To this end, we consider the input signals for actors and media in the following pattern

$$
\begin{aligned}
& \boldsymbol{u}_{i}(t)=\boldsymbol{\Lambda}_{\gamma(i)} \boldsymbol{\chi}_{\gamma(i)}(t)+\sum_{j \in \mathbb{V}_{\gamma(i)}^{a}} a_{i j}\left(\boldsymbol{y}_{j}(t)-\boldsymbol{y}_{i}(t)\right)+b_{i \gamma(i)}\left(\boldsymbol{H}_{\gamma(i)} \boldsymbol{\Pi}_{\gamma(i)} \boldsymbol{\chi}_{\gamma(i)}(t)-\boldsymbol{y}_{i}(t)\right) \\
& \boldsymbol{\mu}_{k}(t)=\sum_{l=1}^{m}\left|c_{k l}\right|\left(\boldsymbol{\eta}_{l}(t) \operatorname{sgn} c_{k l}-\boldsymbol{\eta}_{k}(t)\right)
\end{aligned}
$$

where $\boldsymbol{\Pi}_{\gamma(i)}$ and $\boldsymbol{\Lambda}_{\gamma(i)}$ are solutions to the equations

$$
\begin{aligned}
& \boldsymbol{F}_{\gamma(i)} \boldsymbol{\Pi}_{\gamma(i)}+\boldsymbol{G}_{\gamma(i)} \boldsymbol{\Lambda}_{\gamma(i)}=\boldsymbol{\Pi}_{\gamma(i)} \boldsymbol{\Psi}_{\gamma(i)}, \\
& \boldsymbol{H}_{\gamma(i)} \boldsymbol{\Pi}_{\gamma(i)}=\boldsymbol{\Xi}_{\gamma(i)},
\end{aligned}
$$

for $i=1, \ldots, n$. Next, we begin to examine the convergence behavior of opinion formation in a corrupted social context.

Theorem 4. Consider the entire social network $\mathcal{G}^{e}$ satisfying $\sum_{i=1}^{n} \sum_{k=1}^{m} d_{k i}=0$. If the following conditions hold,

1). media graph $\mathcal{G}^{m}$ is quasi-strongly connected, balanced and structurally balanced;

2). the medium-to-actor couplings are lower bounded away from zero, i.e., $b_{i \gamma(i)}>\epsilon>0$, for all $i \in \mathbb{V}^{a}$; 
3). $\quad \boldsymbol{\Psi}_{k} \sim{ }_{s} \boldsymbol{\Psi}_{l}$ for all $k, l \in \mathbb{V}^{m}$;

4). $\quad \boldsymbol{\Xi}_{k}^{\top} \boldsymbol{\Xi}_{k} \succ 0$, for all $k \in \mathbb{V}^{m}$;

then the control laws (14)-(16) solve the output bipartite synchronization problem for the passive actor systems (7a) with detectable $\left(\boldsymbol{H}_{i}, \boldsymbol{F}_{i}\right)$ for all $i \in \mathbb{V}^{a}$. More importantly, the stably synchronized output trajectories of actors rely entirely on the output of media.

Proof. To promote the analysis, we introduce an auxiliary variable $\boldsymbol{e}_{i}(t)=\boldsymbol{x}_{i}(t)-\boldsymbol{\Pi}_{\gamma(i)} \boldsymbol{\chi}_{\gamma(i)}(t)$ for $i \in \mathbb{V}^{a}$, whose timederivative follows by $(7 a)$ as

$$
\dot{\boldsymbol{e}}_{i}(t)=\left(\boldsymbol{F}_{\gamma(i)}-b_{i \gamma(i)} \boldsymbol{G}_{\gamma(i)} \boldsymbol{H}_{\gamma(i)}\right) \boldsymbol{e}_{i}(t)-\boldsymbol{\Pi}_{\gamma(i)} \boldsymbol{\Theta}_{\gamma(i)} \boldsymbol{\mu}_{\gamma(i)}(t),
$$

where (14)-(16) are taken into account. In equation (17), the first term $\boldsymbol{F}_{\gamma(i)}-b_{i \gamma(i)} \boldsymbol{G}_{\gamma(i)} \boldsymbol{H}_{\gamma(i)}$ on the right-hand side is Hurwitz as a consequence of the passivity assumption and detectability of $\left(\boldsymbol{H}_{i}, \boldsymbol{F}_{i}\right)$. By adopting Corollary 1 to the media network $\mathcal{G}^{m}$, the media states $\chi_{\gamma(i)}$ asymptotically reach the output bipartite synchronization for any non-zero initial conditions, as well as $\boldsymbol{\mu}_{\gamma(i)} \rightarrow \mathbf{0}$ when time goes to infinity. Then, the auxiliary variables $\boldsymbol{e}_{i}$ obeying time evolution rule (17) converge to an asymptotically stable equilibrium state, i.e., $\lim _{t \rightarrow \infty} \boldsymbol{e}_{i}(t) \rightarrow \mathbf{0}$ for all $i \in \mathbb{V}^{a}$. Combining with condition (16), one can immediately obtain that $\lim _{t \rightarrow \infty} \boldsymbol{y}_{i}(t)-\boldsymbol{\eta}_{\gamma(i)}(t)=\mathbf{0}$.

Note that media asymptotically achieve the output bipartite synchronization at their own risk without the involvement of actors. In a more abstract setting, the outputs of media play a virtual role of the external references for the dynamical systems (7a), which are tracked by actors under the control law (14). Therefore, one can conclude that actors asymptotically follow a non-zero synchronization trajectory which does not depend on dynamical properties of actors.

Remark 7. In control theory, equations 15 and 16 resemble Francis equations in the output regulation problem. For this reason, the solvability conditions suggested by [40] are available to examine the existence of matrices $\boldsymbol{\Pi}_{\gamma(i)}$ and $\boldsymbol{\Lambda}_{\gamma(i)}$ such that equations (15) and (16) hold. Theorem 4 claims that members of media networks ignore the ideas of actors while forming opinions. The medium, which produces the reference-synchronization signal, plays the role of an exosystem for actors within its influence scope. Thus, one can apply the internal model principle to media systems by following the classical namesake in the control field |41. By exploring an internal model in media dynamics, we can explicitly derive the analytic expression of equilibrium opinion trajectories of agents. See [42] of internal-model-based synchronization problem on cooperative networks and the references therein for more details.

We now provide some valuable remarks and sociological narratives on the proposed theoretical results. The overarching point is that the influence of information exchanging among actors vanishes in this case. That is, the diffusion-like term $\sum_{j \in \mathbb{V}_{\gamma(i)}^{a}} a_{i j}\left(\boldsymbol{y}_{j}-\boldsymbol{y}_{i}\right)$ in 14 degenerates implicitly to zero since the outputs of actors belonging to the same subgroup become identical with each other, owing to (16). Such a nullification phenomenon of interpersonal communication is commonly seen in the autocracy where self-interested dictators prefer information control for political survival purposes [43]. With the technical revolution in communication, especially the recent penetration of social-networking tools, such as Facebook and Twitter, the autocratic phenomenon can be dramatically mitigated by the enhancement of interpersonal communication [39]. In comparison to the relatively democratic controller (??), the actual opinions (the state variables) of media appear in the opinion protocol 14. As such, the propaganda strategy based on a mix of inner attitudes and expressed ideas allows the media to manipulate public opinions, creating an autocratic circumstance [44].

On the network interconnection structure, condition 2) given in Theorem 4 guarantees that every medium has a direct influence on its own viewers. Moreover, topological constraints, including connectivity and (structural) balance, are imposed only on the media graph, but the explicit knowledge of the interaction relationships among actors is not required. That is to say, the results are valid for any interconnection configuration of actor subgraphs $\mathcal{G}_{\gamma(i)}^{a}$. We even allow the extreme case in which actors do not communicate with one another and only believe-information spread through public media, i.e., $\sum_{i, j=1}^{n} a_{i j}=0$.

\section{Algebraic Graph-Theoretical Interpretations}

In this section, we restrict our attention to the investigation of the underlying interconnection topology of the entire social network from the perspective of algebraic graph theory.

To illustrate the developed ideas in further depth, we conduct the following investigation in the context of Laplacian dynamics

$$
\left[\begin{array}{l}
\dot{\boldsymbol{x}}(t) \\
\dot{\boldsymbol{\chi}}(t)
\end{array}\right]=-\boldsymbol{L}^{e}\left[\begin{array}{l}
\boldsymbol{x}(t) \\
\boldsymbol{\chi}(t)
\end{array}\right],
$$

whereby actors and media are of single-integrator dynamics with state-feedback control laws. Therefore, the modulus (bipartite) output synchronization problem reduces to modulus (bipartite) state consensus problem as typically studied in the majority of publications on opinion formation problems, e.g., [16], [17]. Evidently, the spectral properties of the Laplacian $\boldsymbol{L}^{e}$ are of central importance to characterize the convergence behavior of the closed-loop system (18). In analogy with the analysis of the above section, let actor graphs $\mathcal{G}^{a}$ be strongly connected and media graph $\mathcal{G}^{m}$ be quasi-strongly connected and structurally balanced. At slight different with the generic model investigated in Theorem 2 , the Assumption 3 is relaxed to $\prod_{k=1}^{m} \sum_{i=1}^{n} b_{i k}>0$ and $\prod_{k=1}^{m} \sum_{i=1}^{n} d_{k i}>0$, meaning there exists at least one member in each actor-subgroup 
$\mathbb{V}_{k}^{a}$ connecting to and being connected from the medium $k$ for all $k \in \mathbb{V}^{m}$. This relaxation arises from the fact that the balance precondition can be discarded when the self-dynamics term is absent. With the help of Lemma 1 , the entire graph $\mathcal{G}^{e}$ is quasi-strongly connected and structurally balanced. Hence, the Laplacian $\boldsymbol{L}^{e}$ has a simple zero root, and other non-zero eigenvalues have a positive real part. Moreover, there exist vectors $\boldsymbol{\nu}^{e}:=\left[\nu_{1}^{e}, \ldots, \nu_{n+m}^{e}\right] \in \mathbb{R}^{n+m}$ and $\boldsymbol{\rho}^{e}:=\left[\rho_{1}^{e}, \ldots, \rho_{n+m}^{e}\right] \in\{ \pm 1\}^{n+m}$ such that $\boldsymbol{\nu}^{e} \in \operatorname{ker}\left(\boldsymbol{L}^{e}\right)^{\top}$ and $\boldsymbol{\rho}^{e} \in \operatorname{ker} \boldsymbol{L}^{e}$. In terms of the node classification of $\mathbb{V}^{e}$, these eigenvectors can be decomposed respectively into $\boldsymbol{\nu}^{e}=\left[\left(\boldsymbol{\nu}^{a}\right)^{\top},\left(\boldsymbol{\nu}^{m}\right)^{\top}\right]^{\top}$ and $\boldsymbol{\rho}^{e}=\left[\left(\boldsymbol{\rho}^{a}\right)^{\top},\left(\boldsymbol{\rho}^{m}\right)^{\top}\right]^{\top}$, such that

$$
\left(\boldsymbol{L}^{a}+\boldsymbol{B}\right) \boldsymbol{\rho}^{a}=\tilde{\boldsymbol{B}} \boldsymbol{\rho}^{m}, \quad\left(\boldsymbol{L}^{m}+\boldsymbol{D}\right) \boldsymbol{\rho}^{m}=\tilde{\boldsymbol{D}} \boldsymbol{\rho}^{a} .
$$

According to the properties of SB theory and the decomposition of eigenvector $\boldsymbol{\rho}$, the relations in (19) expose that $\boldsymbol{\rho}^{a} \in$ $\operatorname{ker} \boldsymbol{L}^{a}, \boldsymbol{\rho}^{m} \in \operatorname{ker} \boldsymbol{L}^{m}$, and the entries of $\boldsymbol{\rho}^{a}$ satisfy $\rho_{i}^{a}=\rho_{\gamma(i)}^{m}$ for all $i \in \mathbb{V}^{a}$. In other words, the mode of the attitude (support or oppose) of media is in accordance with actors' within their influence scope.

Following the resutls on Lemma 1 and Lemma 2 in $\left[20\right.$, for any initial conditions $\boldsymbol{x}(0) \in \mathbb{R}^{n}, \chi(0) \in \mathbb{R}^{m}$, the Laplacian dynamics 18 yields that

$$
\begin{aligned}
\lim _{t \rightarrow \infty} \boldsymbol{x}(t) & =\left[\left(\overline{\boldsymbol{\nu}}^{a}\right)^{\top} \boldsymbol{x}(0)+\left(\overline{\boldsymbol{\nu}}^{m}\right)^{\top} \boldsymbol{\chi}(0)\right] \boldsymbol{\rho}^{a}, \\
\lim _{t \rightarrow \infty} \chi(t) & =\left[\left(\overline{\boldsymbol{\nu}}^{a}\right)^{\top} \boldsymbol{x}(0)+\left(\overline{\boldsymbol{\nu}}^{m}\right)^{\top} \boldsymbol{\chi}(0)\right] \boldsymbol{\rho}^{m},
\end{aligned}
$$

where $\overline{\boldsymbol{\nu}}^{a}:=\boldsymbol{\nu}^{a} /\|\boldsymbol{\nu}\|$ and $\overline{\boldsymbol{\nu}}^{m}:=\boldsymbol{\nu}^{m} /\|\boldsymbol{\nu}\|$. Equations in 20 imply that the right-eigenvector $\boldsymbol{\rho}^{e}$ determines to which phase the opinions of agents belong, while normalized left-eigenvector $\overline{\boldsymbol{\nu}}^{e}$ characterizes the total (direct and indirect) relative influence of each agents' initial impressions to the settled outcome of group discussion.

In cooperative networks without negative weighted edges, Perron-Frobenius theorem and (quasi-)strong connectedness of unsigned graphs entail the existence of a unique (non-negative) positive real left-eigenvector of graph Laplacian [45]. When graphs have negative edges, however, there is rarely counterpart result in the existed literature. A primary challenge is that the uniform positiveness (non-negativeness) may not be preserved in a graph with negative weighted edges. The next lemma which makes progress on this problem is quite apparent, and the proof is omitted here.

Lemma 2. Consider a quasi-strongly connected and structurally balanced signed graph $\mathcal{G}$ associated with a Laplacian matrix $\boldsymbol{L} \in$ $\mathbb{R}^{n \times n}$. If there exist vectors $\boldsymbol{\nu}=\left[\nu_{1}, \ldots, \nu_{n}\right]^{\top}$ and $\boldsymbol{\rho}=\left[\rho_{1}, \ldots, \rho_{n}\right]^{\top} \in\{ \pm 1\}^{n}$ such that $\boldsymbol{\nu} \in$ ker $\boldsymbol{L}^{\top}$ and $\boldsymbol{\rho} \in$ ker $\boldsymbol{L}$, then one can obtain

where $\hat{\boldsymbol{L}}$ is the Laplacian of the associated unsigned graph of $\mathcal{G}$.

$$
\hat{\boldsymbol{\nu}}:=\left[\nu_{1} \rho_{1}, \ldots, \nu_{n} \rho_{n}\right]^{\top} \in \operatorname{ker} \hat{\boldsymbol{L}}^{\top}, \quad \text { and }|\boldsymbol{\rho}| \in \operatorname{ker} \hat{\boldsymbol{L}}
$$

Lemma 2 enables us to furnish a signed graph with a counterpart of Perron-Frobenius left eigenvector by searching on its associated unsigned graph. In particular, Kirchhoff's Matrix-Tree theorem [46] provides a constructive way to find the vector $\hat{\boldsymbol{\nu}}^{e}$ in the cokernel of $\boldsymbol{L}^{e}$ as follows: $\hat{\nu}_{p}^{e}:=\nu_{p}^{e} \rho_{p}^{e} \geq 0\left(p \in \nabla^{e}\right)$ is equal to the sum, over all spanning tree rooted at node $p$ in graph $\mathcal{G}\left(\hat{\boldsymbol{L}}^{e}\right)$. In relation to the topological configuration of the entire social network, the condition 3.3). of Lemma 1 means that there exists at least one non-zero entry of $\boldsymbol{\nu}^{a}$ corresponding to the ordinary node that is the root of a spanning tree of graph $\mathcal{G}^{e}$. In other word, actors make a direct contribution to the collective decision making rather than merely media dominating.

In the corruption case with autocratic media where $\sum_{i=1}^{n} \sum_{k=1}^{m} d_{i k}=0$, it is evident that no actor has a spanning tree rooted from it on the social networks $\mathcal{G}^{e}$. Consequently, the counting spanning tree method gives rise to $\rho_{i}^{a} \in\{ \pm 1\}$ and $\nu_{i}^{a} \rho_{i}^{a}=0$ for all $i \in \mathbb{V}^{a}$, i.e., $\boldsymbol{\nu}^{a}=\mathbf{0}$. In reference to the equilibrium opinion vector of (20), the autocratic media control the outcome of collective debates in a corrupt society and ordinary actors eventually forget their initial attitudes.

\section{NUmERICAL ILLUSTRATIONS}

In this section, we provide some numerical tests to qualitatively and quantitatively demonstrate the developed results.

The paradigmatic example of social networks is illustrated in Fig. 2 comprising three media and nine ordinary actors which self-organize into three separate groups. Note that the color configuration of the nodal border is applied correspondingly to colorize their state trajectories.

Clearly, this network structure satisfies the connectedness requirement and is structurally balanced. Namely, the entities of the social network split into two hostile camps. The associated Laplacian matrix has a single eigenvalue 0 , and all other non-zero eigenvalues have a positive real part. Additionally, one can immediately verify $\boldsymbol{\rho}^{e}=[1,1,1,1,1,-1,-1,-1,-1,1,1,-1]^{\top} \in$ ker $\boldsymbol{L}^{e}$, evidencing that the group of actors has the same sign as the medium to which they connect.

In the society with autocratic media, the network decomposition into two opposed subgroups remains unchanged, but one can compute that $\boldsymbol{\nu}^{a}=\mathbf{0}$, agreeing with the analysis developed in Section 4 . That is, ordinary citizens are entitled little power in public opinion discussion and oligarchs or centralized factions possess social dominance exclusively. 
Consider the social entities (actors and media) discuss and form opinions about four issues. To save cliché, let all of input-to-state and state-to-output maps in dynamics (7) and (8) be the identity matrix and let the system matrices be

$$
\begin{aligned}
\boldsymbol{\Psi}_{\mathrm{V} m} & =\left[\begin{array}{cccc}
-2 & 1 & \frac{11}{5} & \frac{9}{25} \\
\frac{3}{2} & -\frac{9}{10} & -\frac{17}{50} & -\frac{7}{50} \\
\frac{1}{2} & -2 & -\frac{3}{2} & \frac{1}{2} \\
-\frac{27}{10} & \frac{19}{10} & \frac{16}{5} & -\frac{3}{5}
\end{array}\right], \boldsymbol{F}_{\mathrm{V}_{1}^{a}}=\left[\begin{array}{cccc}
\frac{1}{2} & -1 & \frac{1}{2} & -\frac{1}{2} \\
2 & -\frac{3}{2} & -1 & 0 \\
\frac{1}{2} & -1 & \frac{1}{2} & -\frac{3}{2} \\
\frac{3}{2} & -\frac{5}{4} & 1 & -\frac{5}{2}
\end{array}\right], \\
\boldsymbol{F}_{\mathbb{V}_{2} a} & =\left[\begin{array}{cccc}
\frac{2}{5} & -\frac{29}{20} & -\frac{1}{2} & \frac{3}{5} \\
4 & -\frac{7}{2} & -3 & 0 \\
-\frac{4}{5} & -\frac{17}{20} & -\frac{1}{2} & \frac{4}{5} \\
0.4 & -\frac{7}{10} & 1 & -\frac{7}{5}
\end{array}\right], \boldsymbol{F}_{\mathbb{V}_{3} a}=\left[\begin{array}{cccc}
\frac{3}{5} & -\frac{37}{20} & -\frac{9}{10} & \frac{7}{10} \\
\frac{5}{2} & -\frac{13}{10} & -\frac{1}{10} & -\frac{7}{5} \\
\frac{5}{2} & -\frac{17}{5} & -\frac{18}{5} & \frac{9}{5} \\
\frac{5}{2} & \frac{21}{10} & \frac{2}{5} & -\frac{29}{10}
\end{array}\right],
\end{aligned}
$$

which commonly have a pair of purely imaginary eigenvalues $\pm \iota$ and the others belong to open left-half complex plane. In particular, the eigenspaces associated with $\pm \iota$ share in common for all social entities in $\mathbb{V}^{e}$. In response to Theorem 2 . the opinions of actors in the network reach polarization under the protocol (??), as shown in Fig. 3(a). From this figure, the opinion polarity is achieved as time evolves, whereas the asymptotic states of agents fall into two opposing stable trajectories rather than a static value. To visualize the opinion neutralization, we set the weight of link $(10,12)$ to be -2 , thus violating the structural balance of graph $\mathcal{G}^{e}$. According to Theorem 3 the public opinions asymptotically turn into neutrality whatever their initial attitudes were. The trajectory curves of opinion variables in the neutralization case are plotted in Fig. 3(b).

In the last test, we modify the system matrices by

$$
\begin{gathered}
\boldsymbol{\Psi}_{\mathbb{V} m}=\left[\begin{array}{cccc}
-\frac{5}{2} & 1 & -\frac{3}{2} & -1 \\
1 & 1 & -1 & -4 \\
\frac{3}{2} & -1 & -\frac{5}{2} & 1 \\
1 & 1 & -1 & -4
\end{array}\right], \boldsymbol{F}_{\mathbb{V}_{1}^{a}}=\left[\begin{array}{cccc}
-1 & 0 & -1 & 0 \\
6 & 2 & 4 & -6 \\
-2 & -2 & -4 & 2 \\
3 & 0 & -1 & -4
\end{array}\right], \\
\boldsymbol{F}_{\mathbb{V}_{2}^{a}}=\left[\begin{array}{cccc}
2 & 3 & -3 & -11 \\
5 & 3 & -5 & -16 \\
-2 & -3 & 1 & 9 \\
3 & 3 & -3 & -12
\end{array}\right], \boldsymbol{F}_{\mathbb{V}_{2}^{a}}=\left[\begin{array}{cccc}
-\frac{7}{2} & 4 & \frac{1}{2} & -4 \\
-1 & 4 & 1 & -6 \\
0.5 & -4 & -\frac{7}{2} & 4 \\
-1 & 4 & 1 & -6
\end{array}\right],
\end{gathered}
$$

whose eigenvalues all have negative real parts except a common semi-simple eigenvalue at zero associated with an isomorphic eigenspace. As discussed in Remark 5, the opinions of actors split into two polarized camps with two stable equilibrium values whose signs are opposite. See Fig. 3(c) for graphic illustration.

\section{Conclusions ANd Future Works}

In this work, we develop a model of opinion dynamics governed by endogenous (self-dynamics) and exogenous (inflowing information) factors. Our focus is to examine how mass media formulate and change public opinions. While our initial results are mathematical, we provide extensive analysis and interpretation to show that the developed opinion protocols align well with what one would expect in a sociological context. By exploring the structural balance in interaction networks, analysis techniques from the $\mathrm{PH}$ system theory provide theoretical results on the formation of agent opinions in the environment of interpersonal chatting and media competition. Aside from the topological requirement on interaction networks, a certain degree of homogeneity among agents is necessary to establish polarization or synchronization in public opinions. We also provide a criterion for the neutralization of opinion dynamics in terms of a sufficient condition. Furthermore, by drawing a connection to the output-regulation problem in control theory, we identify the intrinsic mechanism of opinion
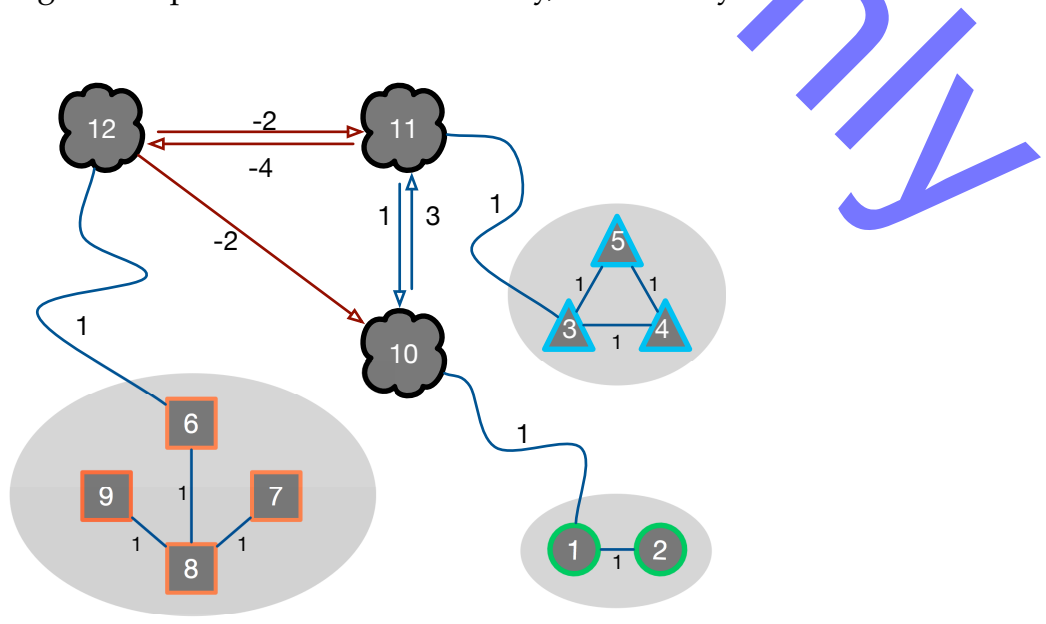

Fig. 2: Paradigmatic social network 


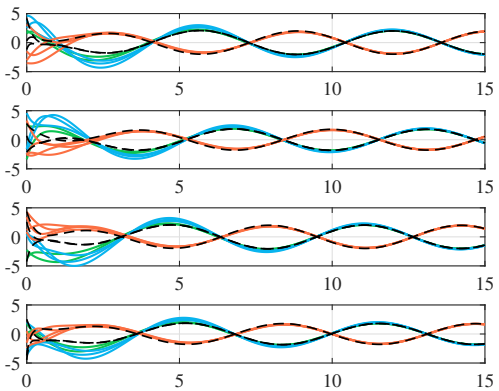

(a) dynamical polarization

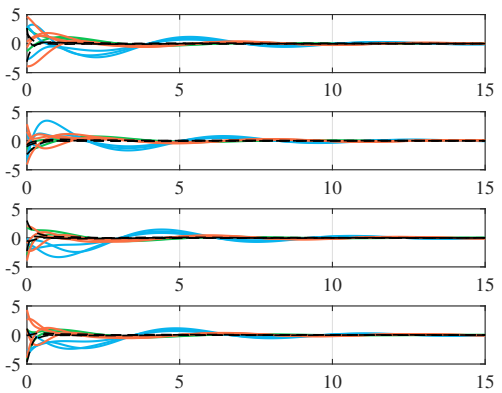

(b) neutralization

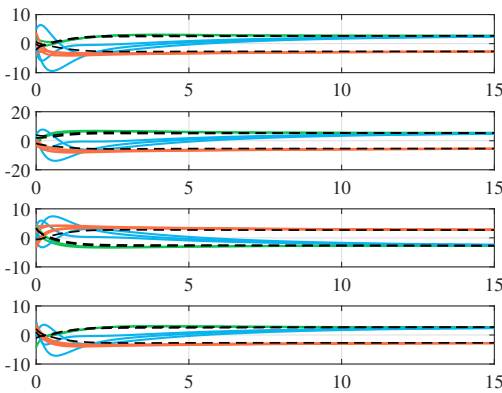

(c) static polarization

Fig. 3: State trajectories of opinion dynamics: $x$-axis is the time scale and $y$-axis is the opinion variables.

manipulation in an autocratic society. Our findings illustrate how ideas from system and control engineering can aid the understanding of opinion formation on real social networks, providing some insights into mathematical sociology.

The directions for future research include the nonlinear extension of opinion dynamics, the time- or state-dependent topology in social interaction, and the communication delay between any pair of individuals in the case that agents can only access others individuals' earlier opinions.

\section{ACKNOWLEDGMENTS}

The authors thank the editors and anonymous reviewers for their excellent comments and suggestions. This work was supported in part by the European Research Council (ERC-CoG-771687) and the Netherlands Organization for Scientific Research (NWO-vidi-14134), in part by the German Research Foundation within the Priority Program SPP 1914 “CyberPhysical Networking", and in part by the joint Sino-German Research Project "Control and optimization for event-triggered networked autonomous multi-agent systems (COVEMAS)", which is funded through the German Research Foundation (DFG) and the National Science Foundation China (NSFC).

\section{REFERENCES}

[1] N. Friedkin, "The problem of social control and coordination of complex systems in sociology: A look at the community cleavage problem," IEEE Control Systems Magazine, vol. 35, no. 3, pp. 40-51, 2015.

[2] A. Proskurnikov and R. Tempo, "A tutorial on modeling and analysis of dynamic social networks. Part I," Annual Review in Control, vol. 43, pp. 65-79, 2017.

[3] - "A tutorial on modeling and analysis of dynamic social networks. Part II," Annual Reviews in Control, vol. 45, pp. 166-190, 2018.

[4] M. Rotoli, G. Russo, and M. di Bernardo, "Stabilizing quorum-sensing networks via noise," vol. 65, no. 5, pp. 647-651, 2018.

[5] T. Danino, O. Mondragón-Palomino, L. Tsimring, and J. Hasty, “A synchronized quorum of genetic clocks," Nature, vol. 463, pp. 326-330, 2010.

[6] G. Russo and J. Slotine, "Global convergence of quorum-sensing networks," Physical Review E, vol. 82, p. $041919,2010$.

[7] S. DellaVigna and E. Kaplan, "The fox news effect: Media bias and voting," vol. 122, pp. 1187-1234, 2007.

[8] N. Friedkin, P. Jia, and F. Bullo, "A theory of the evolution of social power: Natural trajectories of interpersonal influence systems along issue sequences," Sociological Science, vol. 3, pp. 444-472, 2016.

[9] R. Hegselmann and U. Krause, "Opinion dynamics under the influence of radical groups, charismatic leaders, and other constant signals: A simple unifying model," Networks and Heterogeneous Media, vol. 10, no. 3, pp. 477-509, 2015.

[10] A. Mirtabatabaei, P. Jia, and F. Bullo, "Eulerian opinion dynamics with bounded confidence and exogenous inputs," SIAM Journal on Applied Dynamical Systems, vol. 13, no. 1, pp. 425-446, 2014.

[11] G. Russo and R. Shorten, "On common noise-induced synchronization in complex networks with state-dependent noise diffusion processes," vol. 369 , no. 15 , pp. $47-54,2018$.

[12] W. Quattrociocchi, G. Caldarelli, and A. Scala, "Opinion dynamics on interacting networks: Media competition and social influence," Scientific Reports, vol. 4, no. 4938, pp. 1-7, 2014.

[13] R. Olfati-Saber, J. Fax, and R. Murray, “Consensus and cooperation in network multi-agent systems," Proceedings of the IEEE, vol. 95, no. 1, pp. 215-233, 2007.

[14] J. Hu and W. Zheng, "Emergent collective behaviors on coopetition networks," Physica A, vol. 378, pp. 1787-1796, 2014.

[15] C. Toth, "Rationality and irrationality in understanding human behaviors: An evaluation of the methodological consequence of conceptualising irrationality," Journal of Comparative Research in Anthropology and Sociology, vol. 4, no. 1, pp. 85-104, 2013.

[16] C. Altafini, "Consensus problems on networks with antagonistic interactions," IEEE Transactions on Automatic Control, vol. 58, no. 4, pp. 935-946, 2013.

[17] W. Xia, M. Cao, and K. Johansson, "Structural balance and opinion separation in trust-mistrust social networks," IEEE Transactions on Control of Network Systems, vol. 3, no. 1, pp. 46-56, 2016.

[18] K. Young, Handbook of social psychology, 2016.

[19] R. Michels, Political parties: A sociological study of the oligarchical tendencies of modern democracy. Hearsts International Library, 1915.

[20] A. V. Proskurnikov, A. Matveev, and M. Cao, "Opinion dynamics in social networks with hostile camps: Consensus vs. polarization," IEEE Transactions on Automatic Control, vol. 61, no. 6, pp. 1524-1536, 2016.

[21] A. van der Schaft, "Port-Hamiltonian systems theory: An introductory overview," Foundations and Trends in Systems and Control, vol. 1, no. 2-3, pp. 173-378, 2014.

[22] T. Hatanaka, N. Chopra, M. Fujita, and M. Spong, Passivity-based control and estimation in network robotics. Springer, 2015. 
[23] Z. Liu, M. Zhang, A. Saberi, and A. Stoorvogel, "State-synchronization of multi-agent systems via static or adaptive nonlinear dynamic protocols," vol. 95, pp. 316-327, 2018.

[24] G. Russo, F. Wirth, and R. Shorten, "On synchronization in continuous-time networks of nonlinear nodes with state-dependent and degenerate noise diffusion," 2018. [Online]. Available: DOI:10.1109/TAC.2018.2829462

[25] M. Mäs, A. Flache, and D. Helbing, "Individualization as driving force of clustering phenomena in humans," Plos Computational Biology, vol. 6, no. 10, p. e1000959, 2010.

[26] A. Proskurnikov and M. Cao, "Polarization in coopetitive networks of heterogeneous nonlinear agents," in Proceedings of 55th IEEE Conference on Decision and Control (CDC), 2016, pp. 6915-6920.

[27] S. Zhai, "Modulus synchronization in a network of nonlinear systems with antagonistic interactions and switching topologies," vol. 33, pp. 184-193, 2016.

[28] F. Liu, D. Xue, S. Hirche, and M. Buss, "Polarizability, consensusability and neutralizability of opinion dynamics on coopetitive networks," 2018. [Online]. Available: https://doi.org/10.1109/TAC.2018.2879599

[29] M. Girvan and M. Newman, "Community structure in social and biological networks," Proceedings of National Academy of Sciences of the United States of America, vol. 99, no. 12, pp. 7821-7826, 2002.

[30] X. Han, S. Cao, Z. Shen, B. Zhang, W. Wang, R. Cressman, and H. Stanley, "Emergence of communities and diversity in social networks," PNAS, vol. 114, no. 11, pp. 2887-2891, 2017

[31] D. Fiore, G. Russo, and M. di Bernardo, "Exploiting nodes symmetries to control synchronization an consensus patterns in multi-agent systems," vol. 1, no. 2, pp. 364-369, 2017.

[32] M. Golubitsky and I. Stewart, "Recent advances in symmetric and network dynamics," vol. 25, no. 097612, 2015.

[33] N. Leonard, "Multi-agent system dynamics: Bifurcation and behavior of animal groups," vol. 38, pp. 171-183, 2014.

[34] V. Srivastava, J. Moehlis, and F. Bullo, "On bifurcations in nonlinear consensus networks," vol. 21, pp. 875-895, 2011.

[35] L. Boudin and F. Salvarani, Modelling opinion formation by means of kinetic equations. Springer, 2010, pp. 245-270.

[36] S. Asch, Effects of group pressure upon the modification and distortion of judgments. Carnegie Press: Pittsburgh, 1951.

[37] _ "Opinion and social pressure," vol. 193, pp. 31-35, 1955.

[38] D. Lazer, M. Baum, Y. Benkler, A. Berinsky, K. Greenhill, F. Menczer, M. Metzger, B. Nyhan, G. Pennycook, D. Rothschild, M. Schudson, S. Sloman, C. Sunstein, E. Thorson, D. Watts, and J. Zittrain, "The science of fake news," vol. 359, pp. 1094-1096, 2018.

[39] C. Jha and S. Sarangi, "Does social media reduce corruption?" Information Economics and Policy, vol. 39, pp. 60-71, 2017.

[40] H. Trentelman, A. Stoorvogel, and M. Hautus, Control theory for linear systems. Springer, 2001.

[41] B. Francis and W. Wonham, "The internal model principle of control theory," Automatica, vol. 12, pp. 457-465, 1976.

[42] P. Wieland, R. Sepulchre, and F. Allgäwer, "An internal model principle is necessary and sufficient for linear output synchronization," Automatica, vol. 47, pp. 1068-1074, 2011.

[43] H. Hveem and C. Knutsen, Governance and knowledge- Politics of foreign investment, technology and ideas. Taylor \& Francis Group, 2012.

[44] S. Wang, C. Huang, and C. Sun, "Modeling self-perception agents in an opinion dynamics propagation society," vol. 90, pp. 238-248, 2014.

[45] C. Godsil and G. Royle, Algebraic graph theory Springer, 2001.

[46] B. Bollobás, Modern graph theory. Springer, New York, 1998.
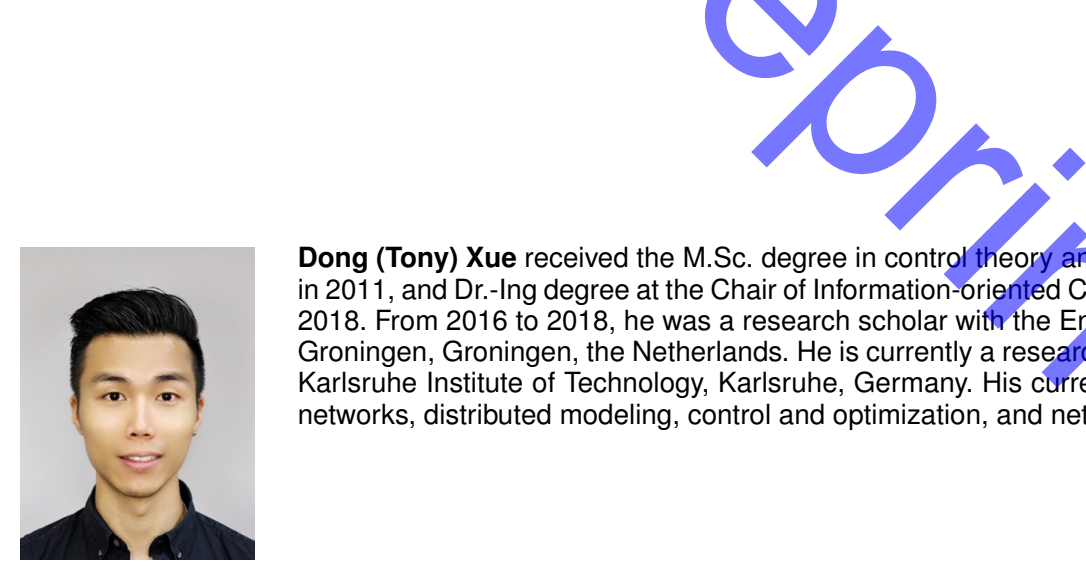

Dong (Tony) Xue received the M.Sc. degree in control theory and engineering from the Tongji University, Shanghai, China, in 2011, and Dr.-Ing degree at the Chair of Information-oriented Control, Technical University of Munich, Munich, Germany, in 2018. From 2016 to 2018, he was a research scholar with the Engineering and Technology Institute (ENTEG), University of Groningen, Groningen, the Netherlands. He is currently a research scientist with the Institute for Applied Computer Science, Karlsruhe Institute of Technology, Karlsruhe, Germany. His current research interests include opinion dynamics and social networks, distributed modeling, control and optimization, and network thermodynamics.

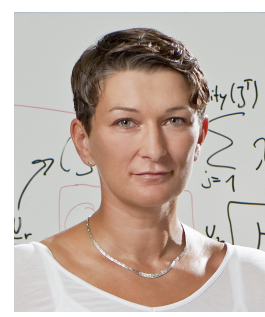

Sandra Hirche holds the TUM Liesel Beckmann Distinguished Professorship and heads the Chair of Information-oriented Control in the Department of Electrical and Computer Engineering at Technical University of Munich, Germany (since 2013). She received the diploma engineer degree in Aeronautical and Aerospace Engineering in 2002 from the Technical University Berlin, Germany, and the Doctor of Engineering degree in Electrical and Computer Engineering in 2005 from Technical University of Munich, Germany. From 2005-2007 she has been a PostDoc fellow of the Japanese Society for the Promotion of Science at the Fujita Laboratory at Tokyo Institute of Technology, Japan. Prior to her present appointment she has been an associate professor at Technical University of Munich. Her main research interests include networked dynamical systems, cooperative and distributed control, and event-triggered control with applications in human-in-the-loop systems, robotics, and infrastructure systems. She has published more than 150 papers in international journals, books, and refereed conferences. Dr. Hirche has served or is serving on the Editorial Boards of the IEEE Transactions on Control Systems Technology and the IEEE Transactions on Haptics. She has received multiple awards such as the Rohde \& Schwarz Award for her Ph.D. thesis in 2005, the IFAC World Congress Best Poster Award in 2005 and together with students Best Paper Awards of IEEE Worldhaptics and IFAC Conference of Manoeuvring and Control of Marine Craft in 2009. 


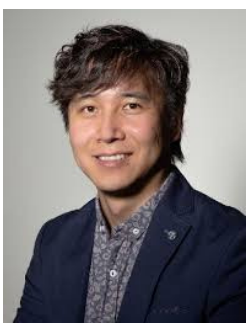

Ming Cao is currently a professor of systems and control with the Engineering and Technology Institute (ENTEG) at the University of Groningen, the Netherlands, where he started as an assistant professor in 2008 . He received the Bachelor degree in 1999 and the Master degree in 2002 from Tsinghua University, Beijing, China, and the Ph.D. degree in 2007 from Yale University, New Haven, CT, USA, all in Electrical Engineering. From September 2007 to August 2008, he was a Postdoctoral Research Associate with the Department of Mechanical and Aerospace Engineering at Princeton University, Princeton, NJ, USA. He worked as a research intern during the summer of 2006 with the Mathematical Sciences Department at the IBM T. J. Watson Research Center, NY, USA. He is the 2017 and inaugural recipient of the Manfred Thoma medal from the International Federation of Automatic Control (IFAC) and the 2016 recipient of the European Control Award sponsored by the European Control Association (EUCA). He is a senior editor of Systems and Control Letters, and an associate editor for IEEE Transactions on Automatic Control, IEEE Transactions on Circuits and Systems and IEEE Circuits and Systems Magazine. He is also the vice chair of the IFAC Technical Committee on Large-Scale Complex Systems and a member of the IFAC Technical Committee on Networked Systems. His research interests include autonomous agents and multi-agent systems, mobile sensor networks and complex networks.

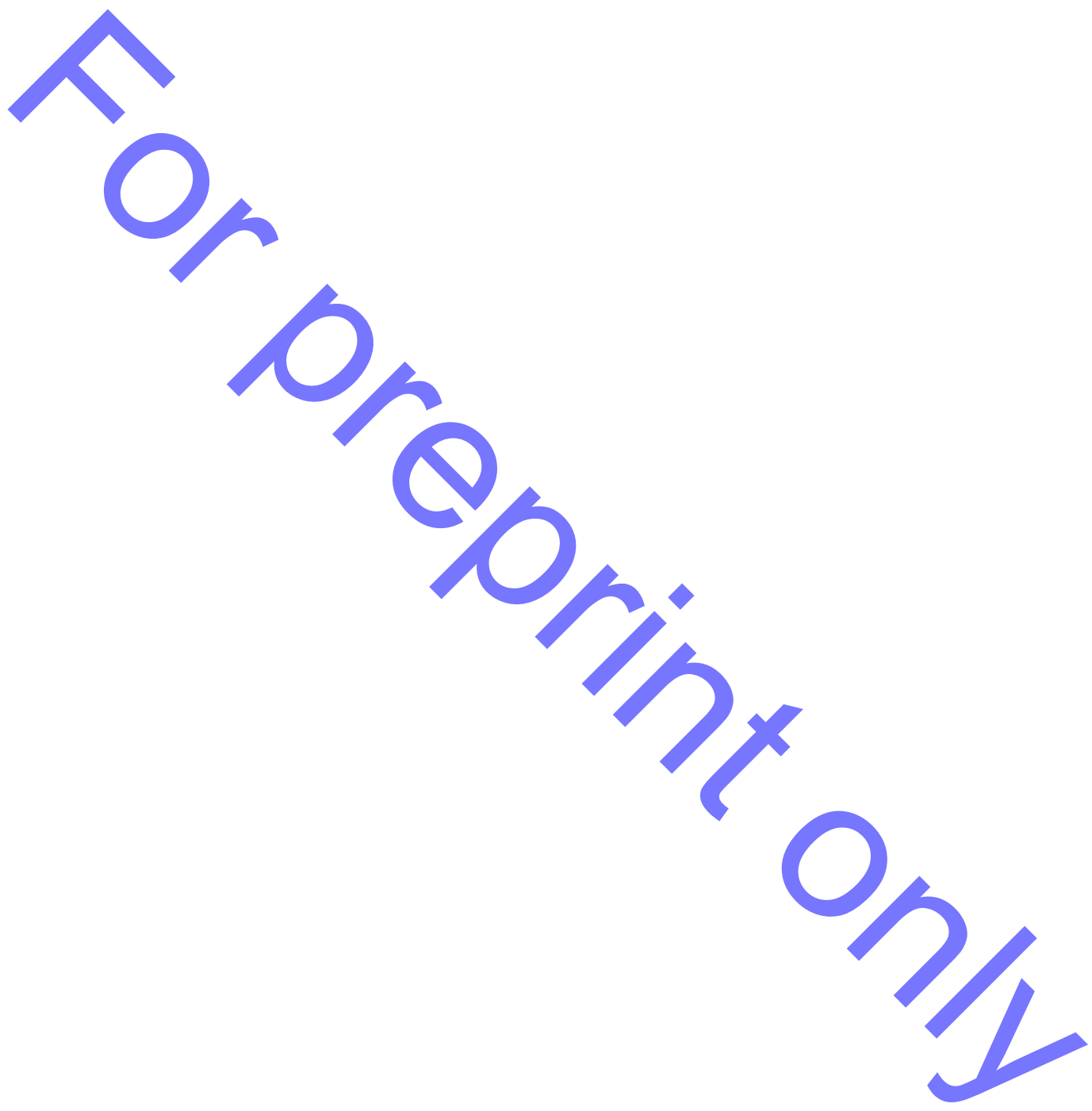

